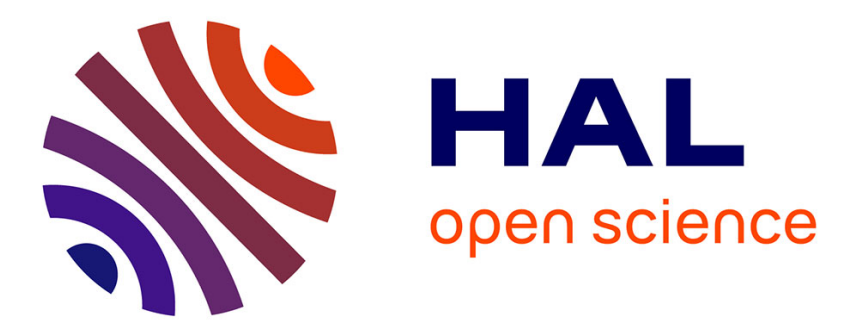

\title{
Pronostic des péritonites à germes résistants aux antibiothérapie(s) de la conférence de consensus de 2000
}

Gilles Arpajou

\section{To cite this version:}

Gilles Arpajou. Pronostic des péritonites à germes résistants aux antibiothérapie(s) de la conférence de consensus de 2000. Médecine humaine et pathologie. 2013. dumas-00915116

\section{HAL Id: dumas-00915116 https://dumas.ccsd.cnrs.fr/dumas-00915116}

Submitted on 6 Dec 2013

HAL is a multi-disciplinary open access archive for the deposit and dissemination of scientific research documents, whether they are published or not. The documents may come from teaching and research institutions in France or abroad, or from public or private research centers.
L'archive ouverte pluridisciplinaire HAL, est destinée au dépôt et à la diffusion de documents scientifiques de niveau recherche, publiés ou non, émanant des établissements d'enseignement et de recherche français ou étrangers, des laboratoires publics ou privés. 
FACULTE MIXTE DE MEDECINE ET DE PHARMACIE DE ROUEN ANNEE 2013

\section{THESE POUR LE DOCTORAT EN MEDECINE}

spécialité : Anesthésie Réanimation

PAR

ARPAJOU Gilles

Né le 27 JUIN 1984 à PARIS

PRESENTE ET SOUTENU PUBLIQUEMENT LE 9 OCTOBRE 2013

\section{PRONOSTIC DES PERITONITES A} GERMES RESISTANTS AUX ANTIBIOTHERAPIE(S) DE LA CONFERENCE DE CONSENSUS DE 2000

PRESIDENT DU JURY : Monsieur le Professeur Bertrand Dureuil DIRECTEUR DE THESE : Monsieur le Docteur Philippe Gouin 
Année Universitaire 2012 - 2013

U.F.R DE MEDECINE - PHARMACIE DE ROUEN

DOYEN :

Professeur Pierre FREGER

ASSESSEURS :

Professeur Michel GUERBET

Professeur Benoît VEBER

Professeur Pascal JOLY

Professeur Bernard PROUST

DOYENS HONORAIRES : Professeurs J. BORDE - Ph. LAURET H. PIGUET - C. THUILLEZ

PROFESSEURS HONORAIRES: MM. M-P AUGUSTIN - J. ANDRIEUGUITRANCOURT - M. BENOZIO - J. BORDE -Ph. BRASSEUR - R. COLIN - E. COMOY - J. DALION - P. DESHAYES - C. FESSARD - J.P FILLASTRE - P. FRIGOT - J. GARNIER - J. HEMET -B. HILLEMAND - G. HUMBERT -J.M JOUANY - R. LAUMONIER - Ph. LAURET - M. LE FUR - J.P. LEMERCIER - J.P. LEMOINE - Melle MAGARD - MM. B. MAITROT - M. MAISONNET - F. MATRAY - P. MITROFANOFF -MME A.M. ORECCHIONI - P. PASQUIS - H. PIGUET - M. SAMSON - MmE SAMSON-DOLLFUS - J.C. SCHRUB - R. SOYER - B. TARDIF - J. TESTART - J.M. THOMINE - C. THUILLEZ - P. TRON - C. WINCKLER - L.M. WOLF 


\section{I- MEDECINE}

\section{PROFESSEURS}

M. Frédéric ANSELME

M. Bruno BACHY

M. Fabrice BAUER

Mme Soumeya BEKRI

M. Jacques BENICHOU

M. Eric BERCOFF

M. Jean-Paul BESSOU

Mme Françoise BEURET-BLANQUART

M. Guy BONMARCHAND

M. Olivier BOYER

M. Jean-François CAILLARD

M. François CARON

M. Philippe CHASSAGNE

M. Alain CRIBIER (surnombre)

M. Antoine CUVELIER

M. Pierre CZERNICHOW

M. Jean-Nicolas DACHER

M. Stéfan DARMONI

communication

M. Pierre DECHELOTTE

Mme Danièle DEHESDIN

M. Philippe DENIS (surnombre)

M. Jean DOUCET

Gériatrie

M. Bernard DUBRAY

M. Philippe DUCROTTE

M. Frank DUJARDIN

M. Fabrice DUPARC

Traumatologique

M. Bertrand DUREUIL

Melle Hélène ELTCHANINOFF

M. Thierry FREBOURG

M. Pierre FREGER

M. Jean-François GEHANNO

Mme Priscille GERARDIN

M. Michel GODIN
$\mathrm{HCN}$ Cardiologie

$\mathrm{HCN}$ Chirurgie Pédiatrique

$\mathrm{HCN}$ Cardiologie

$\mathrm{HCN}$ Biochimie et Biologie Moléculaire

$\mathrm{HCN}$ Biostatistiques et Informatique Médicale

HB Médecine Interne (Gériatrie)

$\mathrm{HCN}$ Chirurgie Thoracique et Cardio-Vasculaire

CPMR Médecine Physique et de Réadaptation

$\mathrm{HCN}$ Réanimation Médicale

UFR Immunologie

HCN Médecine et Santé au Travail

HCN Maladies Infectieuses et Tropicales

HB Médecine Interne (Gériatrie)

$\mathrm{HCN}$ Cardiologie

HB Pneumologie

HCN Epidémiologie, Economie de la Santé

HCN Radiologie et Imagerie Médicale

HCN Informatique Médicale / Techniques de

HCN Nutrition

$\mathrm{HCN}$ Oto-Rhino-Laryngologie

$\mathrm{HCN}$ Physiologie

HB Thérapeutique / Médecine - Interne -

CB Radiothérapie

HCN Hépato - Gastro - Entérologie

HCN Chirurgie Orthopédique - Traumatologique

$\mathrm{HCN}$ Anatomie - Chirurgie Orthopédique et

$\mathrm{HCN}$ Anesthésiologie et Réanimation Chirurgicale

HCN Cardiologie

UFR Génétique

$\mathrm{HCN}$ Anatomie/ Neurochirurgie

$\mathrm{HCN}$ Médecine et Santé au travail

$\mathrm{HCN}$ Pédopsychiatrie

HB Néphrologie 


\begin{tabular}{|c|c|c|}
\hline M. Philippe GRISE & $\mathrm{HCN}$ & Urologie \\
\hline M. Didier HANNEQUIN & $\mathrm{HCN}$ & Neurologie \\
\hline M. Philippe HECKETSWEILLER (surnombre) & $\mathrm{HCN}$ & Hépato-Gastro / Policlinique \\
\hline M. Fabrice JARDIN & $\mathrm{CB}$ & Hématologie \\
\hline M. Luc-Marie JOLY & $\mathrm{HCN}$ & Médecine d'Urgence \\
\hline M. Pascal JOLY & $\mathrm{HCN}$ & Dermatologie - Vénéréologie \\
\hline M. Jean-Marc KUHN & $\mathrm{HB}$ & Endocrinologie et Maladies Métaboliques \\
\hline Mme Annie LAQUERRIERE & $\mathrm{HCN}$ & Anatomie Cytologie Pathologique \\
\hline M. Vincent LAUDENBACH & $\mathrm{HCN}$ & Anesthésie et Réanimation Chirurgicale \\
\hline M. Alain LAVOINNE & UFR & Biochimie et Biologie Moléculaire \\
\hline M. Joël LECHEVALLIER & $\mathrm{HCN}$ & Chrirurgie Infantile \\
\hline M. Patrick LE DOSSEUR & $\mathrm{HCN}$ & Radiopédiatrie \\
\hline M. Hervé LEFEBVRE & $\mathrm{HB}$ & Endocrinologie et Maladies Métaboliques \\
\hline M. Xavier LE LOËT & $\mathrm{HB}$ & Rhumatologie \\
\hline M. Jean-François LEMELAND (surnombre) & $\mathrm{HCN}$ & Bactériologie \\
\hline M. Eric LEREBOURS & $\mathrm{HCN}$ & Nutrition \\
\hline Melle Anne-Marie LEROI & $\mathrm{HCN}$ & Physiologie \\
\hline M. Hervé LEVESQUE & $\mathrm{HB}$ & Médecine Interne \\
\hline Mme Agnès LIARD-ZMUDA & $\mathrm{HCN}$ & Chirurgie Infantile \\
\hline M. Bertrand MACE & $\mathrm{HCN}$ & Histologie, Embryologie, Cytogénétique \\
\hline M. Eric MALLET (surnombre) & $\mathrm{HCN}$ & Pédiatrie \\
\hline M. Christophe MARGUET & $\mathrm{HCN}$ & Pédiatrie \\
\hline Melle Isabelle MARIE & $\mathrm{HB}$ & Médecine Interne \\
\hline M. Jean-Paul MARIE & $\mathrm{HCN}$ & Oto-Rhino-Laryngologie \\
\hline M. LoÏc MARPEAU & $\mathrm{HCN}$ & Gynécologie - Obstétrique \\
\hline M. Stéphane MARRET & $\mathrm{HCN}$ & Pédiatrie \\
\hline M. Pierre MICHEL & $\mathrm{HCN}$ & Hépato-Gastro-Entérologie \\
\hline M. Francis MICHOT & $\mathrm{HCN}$ & Chirurgie Digestive \\
\hline M. Bruno MIHOUT & $\mathrm{HCN}$ & Neurologie \\
\hline M. Pierre-Yves MILLIEZ & $\mathrm{HCN}$ & Chirurgie Plastique, Reconstructrice et \\
\hline \multicolumn{3}{|l|}{ Esthétique } \\
\hline M. Jean-François MUIR & $\mathrm{HB}$ & Pneumologie \\
\hline M. Marc MURAINE & $\mathrm{HCN}$ & Ophtalmologie \\
\hline M. Philippe MUSETTE & $\mathrm{HCN}$ & Dermatologie - Vénéréologie \\
\hline M. Christophe PEILLON & $\mathrm{HCN}$ & Chirurgie Générale \\
\hline M. Jean-Marc PERON & $\mathrm{HCN}$ & Stomatologie et Chirurgie Maxillo-Faciale \\
\hline M. Christian PFISTER & $\mathrm{HCN}$ & Urologie \\
\hline M. Jean-Christophe PLANTIER & $\mathrm{HCN}$ & Bactériologie - Virologie \\
\hline M. Didier PLISSONIER & $\mathrm{HCN}$ & Chirurgie Vasculaire \\
\hline M. Bernard PROUST & $\mathrm{HCN}$ & Médecine Légale \\
\hline
\end{tabular}


M. François PROUST

Mme Nathalie RIVES

la Reproduction

M. Jean-Christophe RICHARD

M. Jean-Christophe SABOURIN

M. Michel SCOTTE

Mme Fabienne TAMION

Melle Florence THIBAUT

M. LUC THIBERVILLE

M. Jacques THIEBOT

M. Christian THUILLEZ

M. Hervé TILLY

M. François TRON

M. Jean-Jacques TUECH

M. Jean-Pierre VANNIER

M. Benoît VEBER

M. Pierre VERA

M. Eric VERSPYCK

M. Olivier VITTECOQ

M. Jacques WEBER
$\mathrm{HCN}$ Neurochirurgie

HCN Biologie et Médecine du Développement et de

HCN Réanimation Médicale, Médecine d'Urgence

HCN Anatomie - Pathologie

$\mathrm{HCN}$ Chirurgie Digestive

HCN Thérapeutique

HCN Psychiatrie d'Adultes

$\mathrm{HCN}$ Pneumologie

$\mathrm{HCN}$ Radiologie et Imagerie Médicale

HB Pharmacologie

CB Hématologie et Transfusion

UFR Immunologie

$\mathrm{HCN}$ Chirurgie Digestive

$\mathrm{HCN}$ Pédiatrie Génétique

$\mathrm{HCN}$ Anesthésiologie et Réanimation Chirurgicale

CB Biophysique et Traitement de l'Image

$\mathrm{HCN}$ Gynécologie - Obstétrique

HB Rhumatologie

$\mathrm{HCN}$ Physiologie

\section{PROFESSEURS ASSOCIES A MI-TEMPS}

\author{
M. Jean-Loup HERMIL \\ M. Alain MERCIER \\ M. Philippe NGUYEN THANH
}

\begin{abstract}
UFR Médecine Générale
UFR Médecine Générale

UFR Médecine Générale
\end{abstract}

\section{MAITRES DE CONFERENCES}

\author{
Mme Noëlle BARBIER-FREBOURG \\ $M$. Jérémy BELLIEN \\ Mme. Carole BRASSE LAGNEL \\ M. Gérard BUCHONNET \\ Mme Mireille CASTANET \\ Mme Sophie CLAEYSSENS \\ M. Moïse COEFFIER \\ M. Vincent COMPERE
}

\author{
HCN Bactériologie - Virologie \\ $\mathrm{HCN}$ Pharmacologie \\ HCN Biochimie \\ HCN Hématologie \\ $\mathrm{HCN}$ Pédiatrie \\ $\mathrm{HCN}$ Biochimie et Biologie Moléculaire \\ HCN Nutrition \\ $\mathrm{HCN}$ Anesthésiologie et Réanimation Chirurgicale
}




$\begin{array}{lll}\text { M. Manuel ETIENNE } & \text { HCN } & \text { Maladies Infectieuses et Tropicales } \\ \text { M. Guillaume GOURCEROL } & \text { HCN } & \text { Physiologie } \\ \text { Mme Catherine HAAS-HUBSCHER } & \text { HCN } & \text { Anesthésie et Réanimation Chirurgicale } \\ \text { M. Serge JACQUOT } & \text { UFR } & \text { Immunologie } \\ \text { M. Joël LADNER } & \text { HCN } & \text { Epidémiologie, Economie de la Santé } \\ \text { M. Jean-Baptiste LATOUCHE } & \text { UFR } & \text { Biologie Cellulaire } \\ \text { Mme Lucie MARECHAL-GUYANT } & \text { HCN } & \text { Neurologie } \\ \text { M. Jean-François MENARD } & \text { HCN } & \text { Biophysique } \\ \text { Mme Muriel QUILLARD } & \text { HCN } & \text { Biochimie et Biologie Moléculaire } \\ \text { M. Vincent RICHARD } & \text { UFR } & \text { Pharmacologie } \\ \text { M. Francis ROUSSEL } & \text { HCN } & \text { Histologie, Embryologie, Cytogénétique } \\ \text { Mme Pascale SAUGIER-VEBER } & \text { HCN } & \text { Génétique } \\ \text { Mme Anne-Claire TOBENAS-DUJARDIN } & \text { HCN } & \text { Anatomie } \\ \text { M. Eric VERIN } & \text { HCN } & \text { Physiologie }\end{array}$

MAITRES DE CONFERENCES ASSOCIES A MI-TEMPS

$\begin{array}{lcl}\text { M. Pierre FAINSILBER } & \text { UFR } & \text { Médecine Générale } \\ \text { M. Emmanuel LEFEBVRE } & \text { UFR } & \text { Médecine Générale } \\ \text { M. Elisabeth MAUVIARD } & \text { UFR } & \text { Médecine Générale }\end{array}$

\section{PROFESSEURS AGREGES OU CERTIFIES}

Mme Dominique LANIEZ

Mme Michèle GUIGOT

d'Expression

\author{
UFR Anglais \\ UFR Sciences Humaines - Techniques
}




\section{II- PHARMACIE}

\section{PROFESSEURS}

$\begin{array}{ll}\text { M. Thierry BESSON } & \text { Chimie Thérapeutique } \\ \text { M. Jean-Jacques BONNET } & \text { Pharmacologie } \\ \text { M. Roland CAPRON } & \text { Biophysique } \\ \text { M. Jean COSTENTIN } & \text { Pharmacologie } \\ \text { Mme Isabelle DUBUS } & \text { Biochimie } \\ \text { M. Loïc FAVENNEC } & \text { Parasitologie } \\ \text { M. Michel GUERBET } & \text { Toxicologie } \\ \text { M. Olivier LAFONT } & \text { Chimie Organique } \\ \text { Mme Isabelle LEROUX } & \text { Physiologie } \\ \text { M. Jean-Louis PONS } & \text { Microbiologie } \\ \text { Mme Elisabeth SEGUIN } & \text { Pharmacognosie } \\ \text { M. Marc VASSE } & \text { Hématologie } \\ \text { M. Jean-Marie VAUGEOIS (Délégation CNRS) } & \text { Pharmacologie } \\ \text { M. Philippe VERITE } & \text { Chimie Analytique }\end{array}$

\section{MAITRES DE CONFERENCES}

Mme Dominique ANDRE

Melle Cécile BARBOT

Mme Dominique BOUCHER

M. Frédéric BOUNOURE

Mme Martine PESTEL-CARON

M. Abdeslam CHAGRAOUI

M. Jean CHASTANG

Mme Marie-Catherine CONCE-CHEMTOB

Mme Elisabeth CHOSSON (Délégation)

Melle Cécile CORBIERE

M. Eric DITTMAR

Mme Nathalie DOURMAP

Melle Isabelle DUBUC

Mme Roseline DUCLOS

M. Abdelhakim ELOMRI

M. François ESTOUR

M. Gilles GARALA
Chimie Analytique

Chimie Générale et Minérale

Pharmacologie

Pharmacie Galénique

Microbiologie

Physiologie

Biomathématiques

Législation Pharmaceutique et Economie de la Santé

Botanique

Biochimie

Biophysique

Pharmacologie

Pharmacologie

Pharmacie Galénique

Pharmacognosie

Chimie Organique

Parasitologie 
Mme Najla GHARBI

Melle Marie-Laure GROULT

M. Hervé HUE

Mme Hong LU

Mme Sabine MENAGER

Mme Christelle MONTEIL

M. Paul MULDER

M. Mohamed SKIBA

Mme Malika SKIBA

Mme Christine THARASSE

M. Rémi VARIN

M. Frédéric ZIEGLER

PROFESSEUR ASSOCIE
Chimie Analytique

Botanique

Biophysique et Mathématiques

Biologie

Chimie Organique

Toxicologie

Science du Médicament

Pharmacie Galénique

Pharmacie Galénique

Chimie Thérapeutique

Pharmacie Hospitalière

Biochimie

Toxicologie

MAITRE DE CONFERENCE ASSOCIE

Mme Sandrine PANCHOU

Pharmacie Officinale

PROFESSEUR AGREGE OU CERTIFIE

Mme Anne-Marie ANZELLOTTI

Anglais

\section{ATTACHES TEMPORAIRES D'ENSEIGNEMENT ET DE RECHERCHE}

Melle. Virginie SEGUIN

Melle. Sophie RABEAU

Melle. Laëtitia LE GOFF
Botanique

Chimie Générale et Minérale

Parasitologie 
CHEF DES SERVICES ADMINISTRATIFS : Mme Véronique DELAFONTAINE

HCN - Hôpital Charles Nicolle

CB - Centre Henri Becquerel

CRMPR - Centre Régional de Médecine Physique et de Réadaptation

HB - Hôpital de Bois Guillaume

CHSR - Centre Hospitalier Spécialisé du Rouvray 


\section{LISTE DES RESPONSABLES DE DISCIPLINES}

Melle Cécile BARBOT

$M$. Thierry BESSON

M. Roland CAPRON

M. Jean CHASTANG

Mme Marie-Catherine CONCE-CHEMTOB

Melle Elisabeth CHOSSON

M. Jean COSTENTIN

Mme Isabelle DUBUS

M. Loïc FAVENNEC

M. Michel GUERBET

M. Olivier LAFONT

M. Jean-Louis PONS

Mme Elisabeth SEGUIN

M. Mohamed SKIBA

M. Marc VASSE

M. Philippe VERITE
Chimie Générale et Minérale

Chimie Thérapeutique

Biophysique

Mathématiques

Législation, Economie de la Santé

Botanique

Pharmacodynamie

Biochimie

Parasitologie

Toxicologie

Chimie Organique

Microbiologie

Pharmacognosie

Pharmacie Galénique

Hématologie

Chimie Analytique

\section{ENSEIGNANTS MONO-APPARTENANTS}

\section{MAITRES DE CONFERENCES}

M. Sahil ADROUCH

Mme Gaëlle BOUGEARD-DENOYELLE

M. Antoine OUVRARD-PASCAUD

PROFESSEURS DES UNIVERSITES

M. Mario TOSI

M. Serguei FETISSOV
Biochimie et Biologie Moléculaire (unité Inserm 905)

Biochimie et Biologie Moléculaire (unité Inserm 614)

Physiologie (Unité Inserm 644)
Biochimie et Biologie Moléculaire (Unité Inserm 614)

Physiologie (Groupe ADEN) 
Par délibération en date du 3 mars 1967, la faculté a arrêté que les opinions émises dans les dissertations qui lui seront présentées doivent être considérées comme propres à leurs auteurs et qu'elle n'entend leur donner aucune approbation ni improbation. 
A Monsieur le Professeur Dureuil,

Cinq ans au sein de votre école, vous m'avez enseigné l'humilité.

Vous me faites l'honneur de présider le jury de cette thèse,

Veuillez trouver dans ce travail,

Le témoignage de mon profond respect et de ma reconnaissance.

A Monsieur le Professeur Veber,

A vos côtés, j'ai appris l'humanité. Vous me faites l'honneur de juger mon travail, Soyez assuré de ma profonde considération.

A Monsieur le Professeur Tuech,

Vous avez accepté de juger cette thèse. Veuillez trouver ici l'expression de mes sincères rémerciements.

A Madame le Docteur Barbier-Frebourg,

Vous avez accepté de juger cette thèse. Veuillez trouver dans ce travail toute ma gratitude.

\section{A Monsieur le Docteur Gouin,}

Tu m'as fait confiance quand je me suis présenté à la porte de ton bureau. Ma volonté de travailler sur un sujet de réanimation était un réel défi pour moi, Je te remercie de m'avoir accompagné dans cette aventure, parfois nocturne. 


\section{REMERCIEMENTS}

$\mathcal{A}$ ma sour, mes parents, mes grands mères, ma famille,

"Si les petits cochons ne te mangent pas, on fera bien quelque chose de toi "

\footnotetext{
${ }^{1}$ Antoine Arpajou, mon père
} 
$\mathcal{A}$ mes Maitres,

Ceux qui ont su me transmettre leur passion de la médecine, être un médecin dans toute sa complexité : un humain, un enseignant, un chercheur ( $\operatorname{Pr}$. Dureuil, Pr. Veber, Pr P.L. Michel, Pr Parc).

$\mathcal{A}$ mon Entraineur de tennis, $\mathcal{M}$. Warmoes, Créateur du concept « nivellement par le haut » : être attiré par l'excellence. J'irai plus loin « n'imite pas l'excellence, crée l'excellence».

$\mathcal{A}$ vous, $\mathcal{M}$. Girault, $\mathcal{M}$. Compère, Pour leur amour de la médecine et de nous le transmettre.

$\mathcal{A}$ mes références, $\mathcal{M}$. Gouín $\mathcal{P}$. , $\mathcal{M}$. Deghmani, Pour leur équilibre personnel et professionnel.

$\mathcal{A}$ Dieppe, Níco $\mathcal{N a t h ~ J C . ~}$

Vous m'aurez appris à être interne d'anesthésie puis à être anesthésiste. Continuez à nous faire aimer ce qui est le plus beau métier. Merci. 
Cette thèse marque aussi 10 ans d'amitiés, de tout horizon

A mes frères et sceurs de Saint Antoine, Brice, Seb, Saimon, Patou, Lionel, Nadir, Xavier, Ciham, Laura, Fatima. Malgré la distance,vous êtes toujours dans mon cœur.

A mes amis, Serge, Johanns, Gauthier, Sinad, Julien, Sophie, Beubeu, Jovani, JC, Rocco. Malgré la distance, vous serez toujours dans mon cœur.

A ma petite famille, Emilie, 4 ans de vie commune (pour moins que ca y en a qui font des bébés...) Charlotte, Laure.

A ma promotion, Juju, Alice, Schéché, Vinz, Laurette, MLT, Zaza, Mimie, Nolwenn, vous êtes magiques.

Aux DESAR de Haute Normandie, continuez à vous ambiancer: Tomtom, Bergis, Yaya, JB, Grognu... Et Boudin.

Je finirais par celle qui détient une place particulière dans mon cœur. Derrière ces 3 mots se dissimulent beaucoup d'images, de sentiments, d'intensités, de rêves... Celle qui m'aura fait vibrer, celle qui nous aura fait vibrer. Vous vous reconnaitrez chacun en elle. Mais où d'un chacun, nous avons uni une équipe,

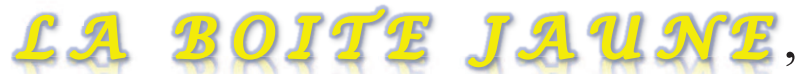

Champion de France de Voile 2011, Vainqueur de régate en 2011, Vainqueur de régate en 2010, Meilleur after 2013, Organisateur en 2012 
"On ne trouve que ce qu'on cherche, on ne cherche que ce qu'on connaît. Il faut travailler, travailler, travailler $\|^{2}$

"Repensez l'impossible » ${ }^{3}$

2 P.L. Michel

3 Tom Cruise 


\section{RESUME}

Introduction : La péritonite aiguë communautaire est une pathologie grave dont les deux axes de traitement principaux sont la chirurgie et l'antibiothérapie, initialement probabiliste. Concernant l'antibiothérapie, les recommandations françaises éditées en 2000 par la Société Française d'Anesthésie Réanimation (SFAR) sous forme d'une conférence de consensus n'ont pas jamais été réactualisées. Depuis 2000, les bactéries résistantes sont en pleine essor, pouvant prendre à défaut les schémas d'antibiothérapie proposés par ce consensus. L'objectif de notre étude était d'évaluer la mobi-mortalité liéee à une antibiothérapie probabiliste inefficace.

Matériel et méthodes : Etude rétrospective monocentrique réalisée au CHU de Rouen, de janvier 2010 à octobre 2012. Nous avons évalué 99 patients atteints d'une péritonite aigue communautaire, qu'ils soient hospitalisés ou non en réanimation. Tous présentaient une résistance bactérienne à l'une des antibiothérapies proposées par le Consensus de 2000. En fonction de l'efficacité de l'antibiothérapie probabiliste prescrite, nous avons constitué un groupe «ATBproba + » $(\mathrm{n}=33)$ et un groupe «ATBproba - » $(\mathrm{n}$ =66). Le critère de jugement principal était la mortalité hospitalière. Nous avons également analysé la morbidité post-opératoire, les bactéries identifiées sur les cultures et leur profil de résistance, la conformité de la prescription de l'antibiothérapie aux recommandations de 2000, l'efficacité théorique des différents schémas d'antibiothérapies proposés par ces recommandations.

Résultats : Les péritonites étaient sus mésocoliques (10\%) et sous mésocoliques (90\%) dont 39\% d'origine appendiculaire. 24\% ont été hospitalisé en réanimation (IGS2 médian 54 [25-96]. La mortalité globale s'élevait à 15\%, un taux de complications de $40 \%$. La durée moyenne de séjour était de $21,3 \pm 30,7$. Le taux de mortalité postopératoire n'était pas différent entre les deux groupes «ATBproba + » et «ATBproba » $(12,1 \%$ vs $16,7 \%, p=0,77)$. De même, le taux de guérison n'était pas significativement différent $(69,7 \%$ vs $54,5 \%, p=0,19)$. Enterococcus sp et Pseudomonas sp étaient surreprésentés par le biais de notre méthodologie. Les protocoles d'antibiothérapie initiale gardaient une bonne sensibilité vis à vis de l'écologie bactérienne digestive retrouvée dans notre série.

Conclusion : Dans notre étude, la morbi-mortalité n'est pas impactée par l'efficacité ou non de l'antibiothérapie initiale. A notre sens, les recommandations de la SFAR de 2000 restent toujours d'actualité.

Mots clef : péritonite secondaire, antibiothérapie probabiliste, sensibilité bactérienne 


\section{SOMMAIRE}

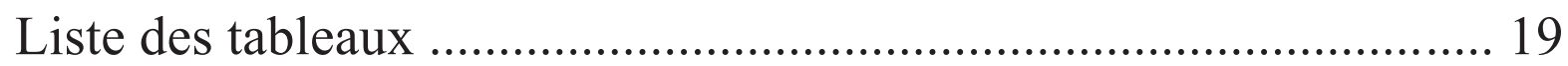

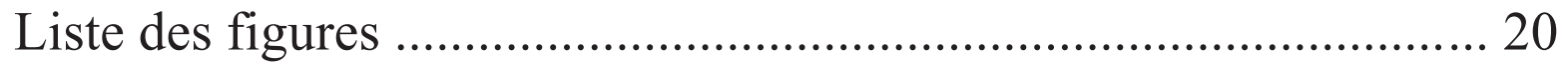

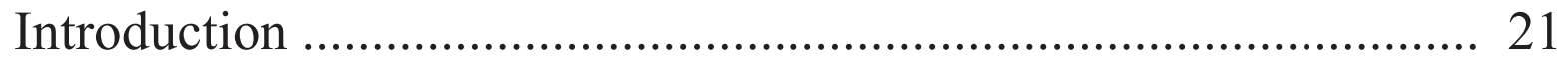

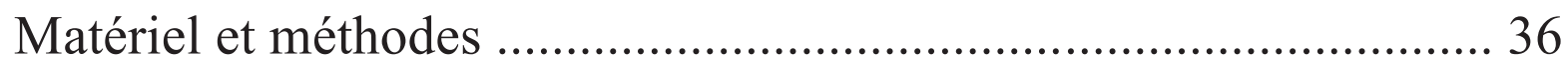

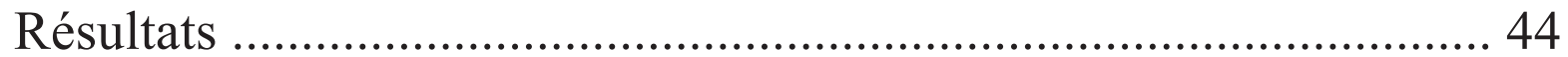

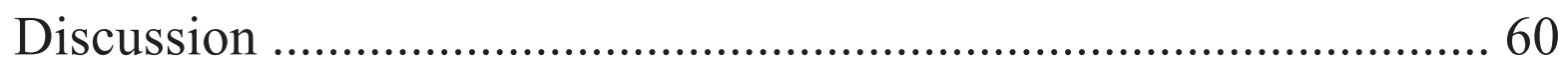

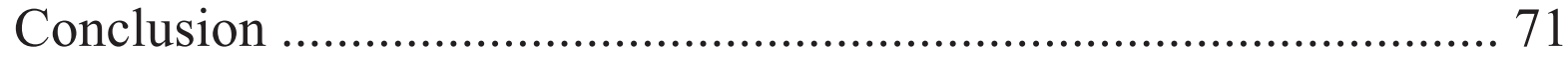

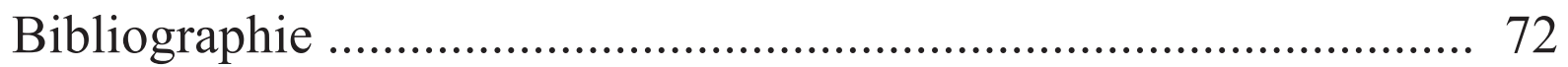




\section{LISTE DES TABLEAUX}

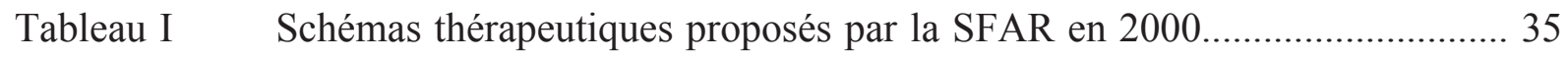

Tableau II Schémas d'antibiothérapies des recommandations américaines................. 35

Tableau III Schémas d'antibiothérapies selon les recommandations européennes......... 36

Tableau IV Critères démographiques de la population et des groupes........................... 46

Tableau V Description des péritonites générales et par groupe....................................47

Tableau VI Mortalité des patients en cas d'antibiothérapie probabiliste non adaptée..... 48

Tableau VII Morbidité des patients en cas d'antibiothérapie probabiliste inadaptée....... 49

Tableau VIII Incidence des bactéries.......................................................................... 51

Tableau IX Profil de sensibilité des bactéries................................................................... 52

Tableau X Nombre de péritonites résistantes à l'antibiothérapie consensuelle.............. 55

Tableau XI Critères démographiques des patients admis en réanimation....................... 58

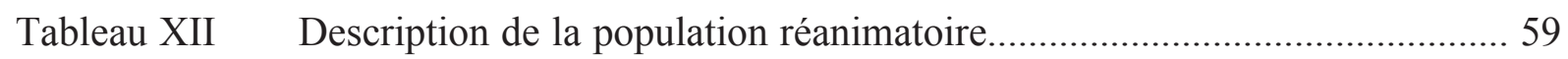

Tableau XIII Guérison et complications en cas de péritonite appendiculaire................... 61 


\section{LISTE DES FIGURES}

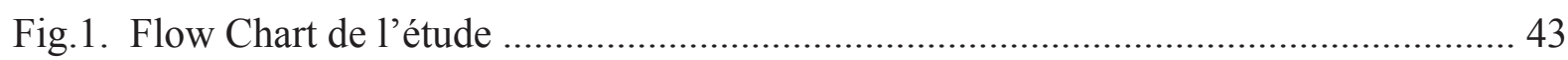

Fig.2. Corrélation entre les résultats de l'examen direct et ceux de la culture...................... 50

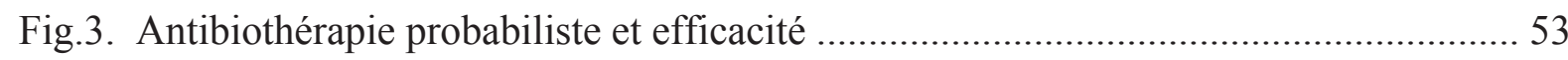

Fig.4. Pourcentage d'antibiothérapie probabiliste administrée recommandée par la ....... 54 conférence de consensus SFAR 2000.

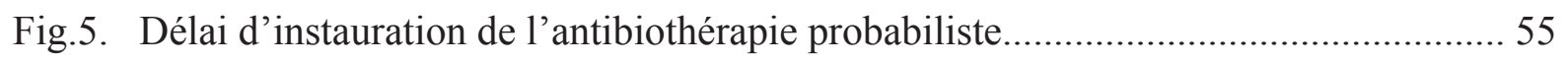

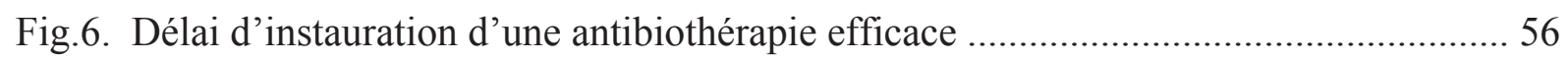

Fig.7. Antibiothérapie réadaptée au sein du groupe « ATBproba - » .................................. 57

Fig.8. Délai de prise en charge chirurgicale ….......................................................... 57

Fig.9. Pourcentage d'antibiothérapie probabiliste efficace en cas de péritonite ............... 60 appendiculaire. 


\section{INTRODUCTION}

La péritonite est une infection aiguë du péritoine. Cette pathologie, fréquente, regroupe plusieurs entités diverses et variées selon le mécanisme et le siège de la lésion. Le traitement s'articule autour de deux axes : le drainage chirurgical et l'antibiothérapie. De plus, dans sa forme grave, cette infection peut nécessiter une prise en charge spécifique en réanimation.

En 2000, une conférence de consensus organisée par la Société Française d'Anesthésie Réanimation (SFAR) avait émis des recommandations sur la prise en charge des péritonites communautaires. Ce texte abordait notamment l'antibiothérapie probabiliste et proposait plusieurs schémas [1].

A ce jour, ces recommandations, qui datent de plus de 10 ans, n'ont pas été réactualisées. Or, au cours de cette période, le profil de résistance des bactéries isolées dans les péritonites communautaire s'est modifié.

Ainsi, il apparaissait nécessaire d'évaluer si, en dépit de l'évolution des résistances, les différents schémas d'antibiothérapies probabilistes proposés étaient encore adéquats, tout en se questionnant sur l'impact réel que peut engendrer une «erreur» d'antibiothérapie probabiliste dans ce cadre bien particulier des péritonites communautaires.

Après quelques rappels d'anatomie et de physiologie du péritoine et du tube digestif, nous aborderons plus en détail les caractéristiques épidémiologiques et physiopathologiques de la péritonite ainsi que ses aspects microbiologiques. Les grandes étapes de prise en charge de cette affection seront développées, tant dans l'étape diagnostique que dans l'étape thérapeutique, avec des rappels sur la prise en charge chirurgicale et médicale, et notamment sur la conduite de l'antibiothérapie.

Pour clore ce préambule, nous rappellerons les différents schémas d'antibiothérapie probabiliste proposés par la Conférence de Consensus française de 2000, ainsi que les recommandations nord-américaines plus récentes $[1,2]$. 


\section{RAPPELS D A ANATOMIE ET DE PHYSIOLOGIE}

\section{a. PERITOINE}

Le péritoine est un double feuillet qui enveloppe l'ensemble des organes du tube digestif. Anatomiquement, il est constitué d'un feuillet viscéral et d'un feuillet pariétal.

Entre ces feuillets, une cavité virtuelle, la cavité péritonéale, est remplie d'un liquide séreux qui permet une mobilité des différentes structures.

Sur le plan histologique, le péritoine est constitué de cellules mésothéliales.

La membrane péritonéale se comporte comme une membrane semi perméable, permettant des mouvements liquidiens dans les deux directions. Un liquide ayant la composition proche d'un ultrafiltrat est secrété, puis réabsorbé dans la circulation générale par le feuillet pariétal. En microscopie électronique, des pores dit stomates permettent la communication entre ces deux domaines [3]. Ils sont localisés dans le territoire du péritoine diaphragmatique. Une partie est réabsorbée dans la circulation lymphatique (30\%) [4].

La mécanique des flux est régie par les mouvements liés au diaphragme, les entrainant sous les coupoles diaphragmatiques. Dans le même temps, les liquides sont aussi soumis à la gravité, les dirigeant dans les zones déclives (cul de sac de Douglas, gouttières paracoliques). Physiologiquement, le liquide péritonéal est stérile, d'un volume de $100 \mathrm{ml}$, pauvre en protéines. Les mouvements diaphragmatiques alliés aux communications avec d'autres circulations font qu'il existe un drainage péritonéal continu.

Andersson and al. a montré sur des rats que la cavité péritonéale ne devait être constituée que d'un ultrafiltat. La présence de bile, de sang total ou de sérum salé entrainait une mortalité variant de 20 à $93 \%$ [5].

Par ailleurs, l'épiploon, structure richement vascularisée, a un rôle essentiel de défense. Il permet aussi un drainage du liquide péritonéal et, en cas d'agression, il colmate les brèches et limite l'infection. D'ailleurs, en cas d'agression, les cellules mésothéliales péritonéales ont une activité secrétrices de médiateurs de l'inflammation [3]. 


\section{b. LE TUBE DIGESTIF}

Le tube digestif recouvert du péritoine, s'étend de l'estomac jusqu'au rectum.

Chaque segment est spécifique par sa structure. Ainsi, chaque segment a une zone de faiblesse.

Histologiquement, la paroi du tube digestif se compose de 5 couches: la muqueuse, la musculo muqueuse, la sous muqueuse, la musculeuse, la tunique conjonctive externe.

L'estomac a deux particularités : une muqueuse divisée en étage « des cryptes » et en étage « des glandes », ainsi qu'une musculeuse composée de 3 couches concentriques.

L'intestin grêle présente une muqueuse à deux étages: les villosités et les glandes de Lieberkühn.

Au niveau colo-rectal, il persiste des cryptes de Lieberkühn et la musculeuse comporte sur sa couche externe par deux bandelettes longitudinales.

La lumière du tube digestif est colonisée par des bactéries dites commensales et des levures constituant une flore saprophyte.

Rappelons qu'à la naissance, le tube digestif du nouveau né est stérile. Ce n'est qu'à l'âge de deux ans environ que l'écologie digestive sera établie.

Les principales bactéries de la flore commensale sont classées en trois groupes :

- les cocci gram positif : Streptococcus sp, Entérococcus sp, Staphylococcus sp,

- les bacilles gram négatif: principalement des entérobactéries (E.coli, entérobactérie groupe KES), parfois des germes non fermentants (Pseudomonas sp, Acinetobacter $s p)$,

- les anaérobies : cocci, bacille (Clostridium sp, Bactéroides sp, Fusobacterium sp).

Il peut exister également des levures, principalement du genre Candida sp.

La composition de la flore varie selon les segments du tube digestif, avec un gradient quantitatif croissant dans le sens oral-anal.

La flore intestinale reste relativement stable chez un même individu. Elle est susceptible de se modifier selon l'alimentation et les antibiotiques [6]. 
Les propriétés chimiques des différents segments du tube digestif induisent une écologie bactérienne différente. Ainsi, l'acidité du liquide gastrique limite la pullulation microbienne. De ce fait, les cocci gram positif sont les plus présents (103). C'est aussi le siège préférentiel du Candida sp.

Au niveau intestinal, le jéjunum contient $10^{\wedge} 4$ germes $/ \mathrm{ml}$ de répartition équitable entre les 3 groupes, cocci Gram positif, bacilles Gram négatif et anaérobies.

Dans le colon, il existe une pullulation microbienne intense, puisque la quantité de bactéries peut atteindre $10^{\wedge} 12$ germes.

Il est à noter qu'en cas d'anomalie du pH gastrique, la flore microbienne est équivalente à la flore iléale ou colique.

La flore microbienne a trois fonctions principales :

- Métaboliques : par la fermentation des résidus alimentaires non digestibles, source d'énergie et de vitamines,

- Trophique sur la muqueuse intestinale,

- De barrière, contre l'implantation de bactéries ayant un pouvoir invasif et pathogène. 


\section{PERITONITE}

\section{a. Définition}

La péritonite est une infection aiguë du péritoine. Sans traitement chirurgical adéquat, le taux de mortalité est de l'ordre de 90\% [7]. Avec l'avènement des antibiotiques, de la chirurgie et de la réanimation, cette mortalité a largement diminué sans pour autant être nulle (15 à 50\%) [8].

L'évolution naturelle d'une péritonite se fait en deux temps : initialement, l'infection diffuse dans l'ensemble du péritoine responsable d'une péritonite généralisée, pouvant se compliquer d'un choc septique. Secondairement, à partir du $5^{\text {ème }}$ jour, elle s'organise plutôt en multiples abcès [9].

\section{b. Siège des péritonites}

Les péritonites sous mésocoliques représentent $70 \%$ des péritonites, les plus fréquentes étant d'origine appendiculaire, puis d'origine colique [10-14].

Chez le sujet agé, la répartition est différente puisque l'origine appendiculaire, sigmoidienne ou biliaire sont à peu près équivalentes ( environ 30\% chacune) [15].

\section{c. Classification}

Devant l'hétérogénéité des péritonites, plusieurs classifications ont été proposées. Elles peuvent être classées selon :

- leur circonstance de survenue (traumatique, occlusion sur brides)

- la localisation anatomique de la perforation digestive :

○ sus mésocolique : estomac, duodénum, voies biliaires

○ sous mésocolique : jéjunum, iléon appendice, colon 
- leur sévérité jugée par des scores :

○ non spécifiques : APACHE 2, IGS 2, utilisés principalement en réanimation

○ spécifiques : Mannheim Peritonitis Index, Peritonitis Index Altona, peu utilisés en pratique $[16,17]$

- l'environnement :

○ communautaire

○ nosocomiale : post opératoire, non post opératoire

La classification la plus utilisée est celle dite de Hambourg, qui organise les péritonites selon leur mode de survenue.

Ainsi, on distingue [18] :

- les péritonites dites primaires: spontanées de l'enfant, spontanées de l'adulte (infection de liquide d'ascite, pneumocoque), secondaires à la dialyse péritonéale, tuberculeuses. Elles sont de traitement médical. Le plus souvent, un seul germe est retrouvé. Elles s'expliquent par une translocation bactérienne ou une dissémination hématogène.

- les péritonites dites secondaires: elles sont les plus fréquentes, secondaire à la perforation d'un organe (perforation, diastatique, sur maladie inflammatoire, d'origine traumatique, post opératoire ou post interventionnelle). Les prélèvements sont généralement pluri-microbiens.

- les péritonites dites tertiaires : péritonites sans germes, fongiques ou à germe ayant un faible pouvoir pathogène.

D'un point de vue pratique, en anesthésie réanimation, on retient davantage la classification selon le siège de la perforation et selon l'environnement, communautaire ou nosocomial.

\section{d. Aspects Microbiologiques}

Par argument de fréquence, les bacilles gram négatif, surtout E.coli, sont les bactéries les plus souvent incriminées (50\%), suivis par les germes anaérobies (16\%) [10]. Plusieurs travaux 
mettent en évidence une émergence de Pseudomonas $s p$, Enterobacter sp, Proteus $s p$, Klebsiella sp respectivement de 2, 4, 6 et $4 \%[10,12]$.

De plus, selon le segment du tube digestif où se trouve la perforation qui contamine le péritoine, les espèces bactériennes et leur répartition peuvent changer. Ainsi, en sus mésocolique, les cocci gram positif, Streptococcus et Enterococcus, et les levures ont une fréquence de l'ordre de 20\% [19].

Les bactéries n'ont pas toutes le même pouvoir pathogène. Les bacilles gram négatif ont un effet très virulent, probablement lié à leur endotoxine. Ils génèrent plus de choc septique, de syndrome de défaillance multi viscérale. A l'inverse, les anaérobies sont plus insidieux. Ils sont responsables d'infection sub-aiguë, larvée, plutôt sous forme d'abcès. Selon certains auteurs, les anaérobies ont une action de copathogène [19]. Ainsi, une étude animale sur un modèle de rat a montré cette différence de pathogénie et de virulence. L'injection d'E.coli en intrapéritonéal entrainait une mortalité de $70 \%$ alors que la co injection d'Enterococcus faecalis et B. Fragilis provoquait certes $100 \%$ d'abcès mais aucune mortalité [20].

\section{e. Epidémiologie des résistances bactériennes.}

Les résistances bactériennes, initialement présentes en milieu hospitalier, sont retrouvées de plus en plus en communautaire. Au cours des 20 dernières années, le profil de résistance des bactéries isolées dans les infections documentées s'est très nettement modifié avec une augmentation des résistances. L'écologie bactérienne a évolué suite à une gestion des antibiotiques probablement moins rigoureuse qu'aujourd'hui, associée à une prescription toujours plus large, une hospitalisation plus fréquente et une vie en institution plus commune [21]. Ces 2 derniers critères émanent, entre autre, d'un vieillissement de la population.

E.coli est naturellement sensible à l'amoxicilline et aux céphalosporines. Aujourd'hui, de nombreux E.coli sont résistants à l'Amox./Ac.clav. et aux Céphalosporine de $3^{\text {ème }}$ génération. De plus, en 2002, la proportion d'E.coli BLSE était de $<1 \%$. Elle passe à moins de $5 \%$ en 2007. Ce fléaux initialement endémique, a maintenant diffusé à l'ensemble de la planète. Les méthodes de prévention, ont permis à partir de 2007, de réduire l'incidence de cette bactérie dans de nombreux pays. Entre 1997 et 2006, huit études ont essayé de démontrer l'émergence de l'E.coli BLSE en communautaire. Les resultats étaient compris entre 13 et $72 \%$. Les études 
étaient non semblables avec des définitions du communautaire très différentes [22]. En 2008, Grobost met en évidence sur des prélèvements urinaires 0,9\% d'E.coli BLSE dont 40 sur 48 présentaient une CTX-M [23]. Chez nos voisins espagnols, le portage intestinal d'E.coli BLSE en communautaire est passé de 0,7 à 5,5\% [24]. De même, des bactéries, principalement communautaires (Salmonella $s p$ ) ont acquis des résistances multiples [25]. Les carbapénémases ont, elles aussi, une augmentation de leur fréquence en ville [26, 27].

\section{f. Diagnostic}

\section{i. $\quad$ Clinique}

Le diagnostic de péritonite est clinique. Il associe une fièvre, des signes digestifs (vomissements, iléus) à une douleur abdominale. Le signe clinique pathognomonique est la contracture. Il existe certaines formes trompeuses: l'état de choc, les syndromes pseudorespiratoires. A noter que le sujet agé présente une forme plus frustre [28].

\section{ii. $\quad$ Paraclinique}

En cas de doute diagnostic, l'abdomen sans préparation peut mettre en évidence un pneumopéritoine, souvent associé à des niveaux hydro-aériques dans le cadre d'un syndrome occlusif.

Cepenant, l'examen de référence reste la tomodensitométrie : elle permet le diagnostic et précise l'étiologie.

Il n'existe pas de place pour l'échographie. Le diagnostic en est difficile et la grande disponibilité aujourd'hui du TDM limite l'intérêt de celle ci [29].

En parallèle, les explorations biologiques montrent un syndrome inflammatoire majeur, associant une hyperleucocytose, une élévation de la CRP et de la PCT [30-33].

\section{iii. Documentation microbiologique}

La documentation microbiologique des péritonites se réalisent par les hémocultures et l'examen du liquide péritonéal qui mettent en évidence la présence d'un ou de plusieurs 
germes. Il est intéressant de noter que la société américaine ne recommande plus les hémocultures systématiques [2].

L'examen bactériologique du liquide péritonéal donne chronologiquement deux informations successives. Dans un premier temps, l'examen microscopique direct délivre un résultat semi quantitatif et peut orienter le spectre antibactérien. Ensuite, une fois la culture réalisée, on procède à l'identification bactérienne et à la réalisation de l'antibiogramme.

La culture recherchera la présence de germes aérobies, anaérobies ainsi que la présence de levures.

L'examen bactériologique est un temps fondamental: il nous permet d'adapter secondairement, le plus souvent sous $48 \mathrm{~h}$, le traitement antibiotique : soit de rétrocéder en rétrécissant le spectre, soit, au contraire, d'élargir le spectre en cas de présence de bactéries particulièrement multi résistantes.

Pour réduire le délai d'obtention des résultats, un antibiogramme direct est couramment pratiqué. L'ensemencement direct du liquide péritonéal permet de donner une bonne orientation des résistances bactériennes, suffisante dans une majorité de péritonites communautaires $[34,35]$. En revanche, en cas de péritonite nosocomiale ou de forme grave de péritonite communautaire (sepsis sévère, choc septique), des antibiogrammes complets seront réalisés, espèce par espéce.

\section{g. Pronostic}

La mortalité des péritonites communautaires varie de 0 à $50 \%$ suivant les séries [36]. Cette donnée est difficilement évaluable de part la grande hétérogénéité possible des péritonites.

Le pronostic dépend :

- du terrain: âge avancé, défaillance d'organe, dénutrition, immunosuppression, comorbidités, cancer,

- du délai de prise en charge chirurgicale [37],

- de la possibilité ou non de réaliser un geste chirurgical optimal [7, 38],

- de l'étiologie : péritonite biliaire augmente la mortalité [39],

- du délai de mise en place d'une antibiothérapie efficace [40], 
- des espèces bactériennes isolées et de leur profil de résistance.

\section{h. Traitement}

Une péritonite est une urgence. Le traitement se décompose en deux axes qui devront être simultanés : chirurgical et médical.

\section{i. Temps chirurgical}

Dès le diagnostic établit, dans un délai le plus court, l'urgence est opératoire.

La chirurgie permet :

- de confirmer le diagnostic,

- de réaliser des prélèvements à visée microbiologique,

- de traiter la cause,

- de limiter les complications ultérieures par le lavage péritonéal abondant. L'association du lavage à un antiseptique ou un antibiotique n'a jamais fait la preuve de son efficacité [1],

- d'optimiser l'hémostase pour limiter les hématomes surinfectés,

- de prévenir les récidives.

La qualité du geste chirurgical est majeure. Seiler, en 2000, démontre une surmortalité de 14\% en l'absence de geste chirurgical satisfaisant [7]. De même, en 1986, Carlet précise une mortalité de $90 \%$ en cas de geste non satisfaisant malgré une antibiothérapie adaptée (versus $6 \%$ en acs de geste optimal) [41].

Les techniques chirurgicales sont diverses selon la cause. D'une suture directe sur une perforation gastrique, jusqu'à une exérèse sans rétablissement de continuité avec double stomie d'amont dans les cas coliques compliqués.

Le traitement de la cause est primordial, les manœuvres de réanimation devront être réalisées simultanément pour ne pas retarder la chirurgie [2, 37, 42, 43]. 


\section{ii. Temps médical}

Le médecin anesthésiste réanimateur évalue le retentissement général de la péritonite. En effet, le patient peut présenter un sepsis sévère, plus ou moins compliqué de choc septique en cas de non réponse à un remplissage vasculaire pour optimiser l'état hémodynamique. Le médecin doit donc évaluer le rententissement sur les différents organes (état respiratoire, fonction rénale, fonction hépatique, entre autres) et sur l'hémostase. Par ailleurs, une péritonite localisée peut faire décompenser une pathologie sous jacente, nécessitant de ce fait une prise en charge réanimatoire spécialisée.

Le traitement chirurgical ne peut se réaliser que sous anesthésie générale. Le médecin anesthésiste conduira l'induction anesthésique la plus adaptée à l'état physiologique du patient. Aussi, dans sa stratégie thérapeutique, il devra introduire une antibiothérapie probabiliste dans les meilleurs délais. Habituellement, les antibiotiques sont administrés dès les prélèvements per opératoires réalisés. Dans les cas les plus graves, cette antibiothérapie est délivrée dès le diagnostic posé.

Les principes de choix de l'antibiothérapie respectent 3 règles :

a) L'antibiothérapie probabiliste doit être adaptée au spectre bactérien et au profil de résistance.

L'objectif de l'antibiothérapie est de couvrir les germes les plus fréquemment retrouvés soit les entérobactéries et les anaérobies [44].

Plus le sepsis est grave, plus le spectre doit être large afin de limiter les trous thérapeutiques, tout retard d'efficacité majorant la morbimortalité [40, 45].

Néanmoins, deux espèces particulières, Enteroccus $s p$ et Candida sp, sont deux germes dont leur rôle reste très discuté dans les péritonites communautaires non graves.

La présence d'un Entérococcus sp non couvert par l'antibiothérapie de référence peut évoluer vers un échec thérapeutique [46, 47].

Dans 3 à $5 \%$, un Candida $s p$ est retrouvé à la culture et l'absence de traitement de ce germe n'entraine pas, dans la majorité des cas, une surmortalité [48].

Les études tendent à montrer qu'un traitement anti-fongique est nécessaire :

- si l'examen direct est positif à levures,

- si le seul germe retrouvé est un candida, 
- s'il existe une candidémie

- $\quad$ si le patient est immunodéprimé [49].

Par contre, Enteroccus sp et Candida sp doivent être couvert en cas de forme grave.

b) La diffusion sur site efficace

Globalement, le péritoine est une membrane qui laisse diffuser correctement les antibiotiques $[50,51]$.

Mais l'effet inoculum important associé à l'acidose locale et à la présence de débris cellulaire entravent la diffusion des antibiotiques. L'impact de la chirurgie permet de rétablir leur efficacité en supprimant ces conditions et notamment en diminuant l'inoculum [52].

Ainsi, presque toutes les classes d'antibiotiques peuvent être utilisées.

c) Introduction précoce d'une antibiothérapie.

En cas de sepsis sévère ou choc spetique, les antibiotiques seront administrés dès la suspicion diagnostique sans attendre la réalisation des prélèvements. En effet, dans les péritonites, l'inoculum est si important que la première dose d'antibiotique ne négativera pas les prélèvements. Cette augmentation de mortalité induite par le retard thérapeutique est très largement démontrée [2, 53]. D'ailleurs, l'objectif d'un traitement antibiotique précoce s'intégrant dans une prise en charge agressive et efficace est un des principes du « surviving sepsis campaign ». , 


\section{LES CONSENSUS D'ANTIBIOTHERAPIES PROBABILISTES}

a. Recommandations françaises

En France, le consensus actuel a été établi par la SFAR en 2000. Il n'a pas été réactualisé depuis.

Cette conférence propose 7 schémas d'antibiothérapie probabiliste possibles pour traiter les péritonites communautaires [1] :

Tableau I Schémas thérapeutiques proposés par la SFAR en 2000

\begin{tabular}{|c|}
\hline Amoxicilline / acide clavulanique + gentamicine ou tobramycine \\
\hline Ticarcilline / acide clavulanique + gentamicine ou tobramycine \\
\hline Cefoxitine \\
\hline Cefotaxime ou ceftriaxone + imidazolé \\
\hline Aminoside + imidazolé \\
\hline Céfépime + imidazolé \\
\hline Pipéracilline + tazobactam à réserver aux formes graves
\end{tabular}

La durée préconisée est de 2 à 10 jours selon le type de péritonite [1].

b. Recommandations nord-américaines

En 2010, les recommandations américaines séparent les péritonites peu et très sévère [2]. Tableau II Schémas d'antibiothérapies des recommandations américaines

\begin{tabular}{|c|c|c|}
\hline & Péritonites peu sévères & Péritonites très sévères \\
\hline Monothérapie & $\begin{array}{c}\text { Tazocilline, Ertapenem, } \\
\text { Moxifloxacine ou } \\
\text { Tygécycline }\end{array}$ & $\begin{array}{c}\text { Imipeneme-cilastatin, } \\
\text { Meropeneme, Piperacilline- } \\
\text { Tazobactam, Doripeneme }\end{array}$ \\
\hline Bithérapie & $\begin{array}{l}\mathrm{C} 2 \mathrm{G} / \mathrm{C} 3 \mathrm{G} / \text { fluoroquinolone } \\
\text { associé au Métronidazole }\end{array}$ & $\begin{array}{c}\text { Cefepime, Ceftazidime, } \\
\text { Ciprofloxacine, ou } \\
\text { Levofloxacine, associé au } \\
\text { Metronidazole }\end{array}$ \\
\hline
\end{tabular}


En France, les C2G sont utilisées en antibioprophylaxie. L'extrapolation des recommandations américaines n'est pas envisageable.

La société américaine ne recommande pas l'adjonction d'aminoside quelque soit le contexte ou la sévérité. En France, il n'est pas recommandé de traiter par fluoroquinolone seul afin de limiter l'émergence rapide de mutants résistants.

c. Recommandations européennes

En 2011, certains experts européens a statué sur l'antibiothérapie probabiliste en cas de péritonite communautaire [54].

Tableau III Schémas d'antibiothérapies selon les recommandations européennes.

\begin{tabular}{|cc|}
\hline Peritonites localisées & Péritonites généralisées \\
\hline Cephalosporine $2 \mathrm{G} / 3 \mathrm{G}+$ Metronidazole & Cephalosporine $3 \mathrm{G}+$ Metronidazole \\
Amoxicilline/Ac.clav & Amoxicilline/Ac.clav \\
Ciprofloxacine + Metronidazole & Imipenem-cilastatin \\
Meropenem \\
Doripenem \\
Ertapenem \\
Moxifloxacin \\
Tigecycline \\
Cefepime \\
Ciprofloxacine + Metronidazole \\
Levofloxacine + Metronidazole
\end{tabular}




\section{JUSTIFICATION DE L'ETUDE}

Le choix d'une antibiothérapie dans un contexte de sepsis sévère doit être raisonné, ce d'autant plus qu'il s'agit d'une antibiothérapie probabiliste.

Le spectre ne doit pas être trop restreint sous peine que l'antibiothérapie soit inactive, aux conséquences parfois fatales. A l'inverse, un spectre trop large génèrerait à long terme des résistances bactériennes, perturbant l'écologie du service et dont on connaît les catastrophes médico-économiques.

Forcé de constater que dans notre expérience, certaines péritonites ne recevaient pas une antibiothérapie efficace mais évoluaient vers la guérison. De plus, les bactéries retrouvées lors des prélèvements avaient modifié leur profil de résistance. De ce fait, certains praticiens ont introduit de nouveaux protocoles antibiotiques « exotiques », sans aucun consensus local.

L'objectif principal de notre étude était d'évaluer la morbi-mortalité des patients présentant une péritonite communautaire à germes résistants aux antibiothérapie(s) recommandée(s) par le consensus de 2000.

A travers ce travail, nous avons souhaité évaluer également la prise en charge globale d'une péritonite aiguë communautaire au CHU de Rouen, cet aspect s'intégrant dans une démarche qualité. 


\section{Matériel et méthodes}

\section{Design de l'étude}

Nous avons réalisé une étude descriptive rétrospective non randomisée, monocentrique au CHU de Rouen, sur une période allant de janvier 2010 à octobre 2012.

Aucune autorisation éthique n'a été nécessaire devant le caractère strictement descriptif et rétrospectif du projet.

\section{Stratégie thérapeutique au CHU de Rouen}

\section{a. Temps chirurgical}

La plupart des diagnostics de péritonites ont été posés dans le service des Urgences du CHU, rarement dans les unités de réanimation. Une fois le diagnostic de péritonite retenu (clinique, imagerie, biologie), le dossier était présenté à l'équipe de chirurgie viscérale et le patient était pris en charge au bloc opératoire dans un délai plus ou moins bref selon l'état d'urgence (sepsis sévère, état de choc), selon l'étiologie et selon la disponibilité du bloc d'urgence en garde (2 à 3 salles).

Le chirurgien a réalisé une voie d'abord (laparotomie ou coelioscopie) adaptée au type de péritonite et au siège suspecté de la perforation, le cas échéant. Les prélèvements bactériologiques étaient réalisés dès l'ouverture du péritoine. Ces prélèvements étaient conservés au réfrigérateur jusqu'au lendemain matin, où ils étaient transportés à l'Institut Biologie Clinique (IBC), dans le service Microbiologie secteur Bactériologie. En cas d'admission en réanimation en post opératoire, les prélèvements étaient acheminés au laboratoire dès la phase opératoire.

\section{b. Temps Anesthésique}

Le médecin anesthésiste réalisait une anesthésie générale adaptée à l'état du patient et fonction de l'évaluation pré opératoire. Si elles étaient nécessaires, les premières mesures de réanimation étaient débutées dès le pré opératoire ou le per opératoire : remplissage 
vasculaire, oxygénothérapie, soutien hémodynamique par amines vasopressives. Aucune antibioprophylaxie n'était réalisée.

Habituellement, l'antibiothérapie probabiliste était administrée dès les prélèvements bactériologies réalisés (liquide péritonéal, hémocultures). En cas de sepsis sévère ou choc septique et en cas de chirurgie différée pour bloc opératoire non disponible immédiatement, les antibiotiques pouvaient être délivrés dès le diagnostic posé.

Le CHU de Rouen ne disposait pas de «protocole» local d'antibiothérapie pour les péritonites. Le choix des antibiotiques était laissé à la discrétion de chaque Médecin Anesthésiste Réanimateur (MAR), d'ordinaire insipiré du consensus de 2000.

\section{c. Temps microbiologique}

\section{i. Bactéries aérobies et anaérobies}

Le liquide péritonéal était acheminé dans un flacon stérile (+/- Portagerm : milieu de transport respectant l'anaérobiose), dès réception du prélèvement :

- L'examen direct était effectué sur le prélèvement après une coloration de Gram. Cette étape permettait de détecter la présence de bacilles ou de cocci, Gram + ou Gram - et de détecter la présence ou non de levures ou de filaments mycéliens.

- La mise en culture du prélèvement de liquide péritonéal était effectuée sur :

$\checkmark$ un milieu chromogène type CPS (milieu solide),

$\checkmark$ une gélose au sang (milieu solide),

$\checkmark$ un milieu liquide (milieu Schaedler), à $37^{\circ}$ pendant $24 \mathrm{~h}$

$\checkmark$ une gélose SCS (milieu solide), placée à $37^{\circ} \mathrm{C}$ en enceinte anaérobie.

- En cas d'examen direct positif, un antibiogramme direct était réalisé. Il se faisait sur un milieu chromogène type CPS et 5 antibiotiques étaient testés :

- ertapénème,

- céfotaxime,

- amoxicilline + acide clavulanique, 
- pipéracilline + tazobactam,

- ceftazidime.

La pastille d'amox/ac clav devait être placée au centre. Ceci permettait de détecter la présence d'une bactérie secrétrice de bétalactamase à spectre élargi (BLSE) par la mise en évidence d'une synergie entre l'amoxicilline/acide clavulanique et la ceftazidime.

L'incubation se faisait en aérobie à $37^{\circ} \mathrm{C}$ pendant $24 \mathrm{~h}$.

- A 24h, un « déblayage » des milieux ensemencés était réalisé.

- A 48h, la lecture des différents milieux permettait les identifications bactériennes complètes.

Si un antibiogramme complet était nécessaire (notamment pour les patients de réanimation), celui-ci se faisait par la technique de diffusion en milieu gélosé (ou technique des disques) sur un milieu Mueller Hinton, incubé pendant 18 à $24 \mathrm{~h}$ à $37^{\circ} \mathrm{C}$, en aérobie.

Les antibiotiques testés variaient en fonction du germe identifié et des recommandations du Comité de l'Antibiogramme de la Société Française de Microbiologie (CA-SFM).

\section{ii. Levures et champignons}

Le liquide péritonéal pouvait également être transmis au laboratoire de mycologie, notamment en cas d'examen direct mettant en évidence des éléments fongiques et pour les patients hospitalisés en réanimation. On y a effectué :

$\checkmark$ Un examen direct après coloration au calcofluor (observation au microscope à fluorescence),

$\checkmark$ Une mise en culture sur un milieu chromogène type CAN2 et sur un milieu Sabouraud enrichi en gentamicine, placés à $30^{\circ} \mathrm{C}$. 
3. Constitution des échantillons

a. Critères d'inclusion

Pouvaient être inclus dans l'étude tous les patients présentant

- Une péritonite aiguë communautaire, définie par

○ un syndrome abdominal aigu,

- débutant en ville ou lors d'une hospitalisation inférieure à $48 \mathrm{~h}$,

- nécessitant un traitement chirurgical,

- Une culture du liquide péritonéal positive,

- Une résitance à l'une des molécules proposée par la conférence de Concensus de 2000:

$\checkmark$ Amoxicilline / acide clavulanique

$\checkmark$ Gentamicine ou tobramycine

$\checkmark$ Ticarcilline / acide clavulanique

$\checkmark$ Cefoxitine

$\checkmark$ Cefotaxime ou ceftriaxone

$\checkmark$ Imidazolé

$\checkmark$ Céfépime

$\checkmark$ Pipéracilline + tazobactam

b. Critères d'exclusion

Les critères d'exclusion étaient

- L'âge inférieur à 18 ans,

- Un syndrome abdominal aigu se déclarant 48h après une admission hospitalière,

- Une péritonite post opératoire immédiate,

- Les infections sur KT de dialyse péritonéale,

- Les infections de liquide d'ascite,

- Les péritonites communautaires non documentées microbiologiquement,

- Les péritonites secondaire à une perforation traumatique. 
c. Sélection des dossiers (flow chart)

Sur une période 34 mois, l'ensemble des prélèvements péritonéaux positifs recensés à l'Institut de Biologie Clinique ont été analysés, soit 2181 antibiogrammes, direct ou sur culture.

Seules les bactéries qui présentaient une résistance (intermédiaire ou complète) à l'une des antibiothérapies de la conférence de consensus de 2000 ont été retenues. Ainsi, 397 patients ont été sélectionnés. Les patients mineurs ont été éliminés.

Dans un second temps, nous avons recherché pour chaque patient sélectionné si la péritonite relevait d'une péritonite aiguë communautaire ou non via son dossier informatique CDP2, logiciel de gestion du dossier médical des patients au CHU de Rouen.

Cent vingt neuf patients répondaient à l'ensemble de nos critères.

Dans un troisième temps, pour chacun de ces patients, le dossier médical «papier »a été consulté pour confirmer les critères d'inclusion et de non-exclusion et recueillir les informations non disponibles sur le dossier informatique.

Au total, 99 dossiers ont été retenus. 


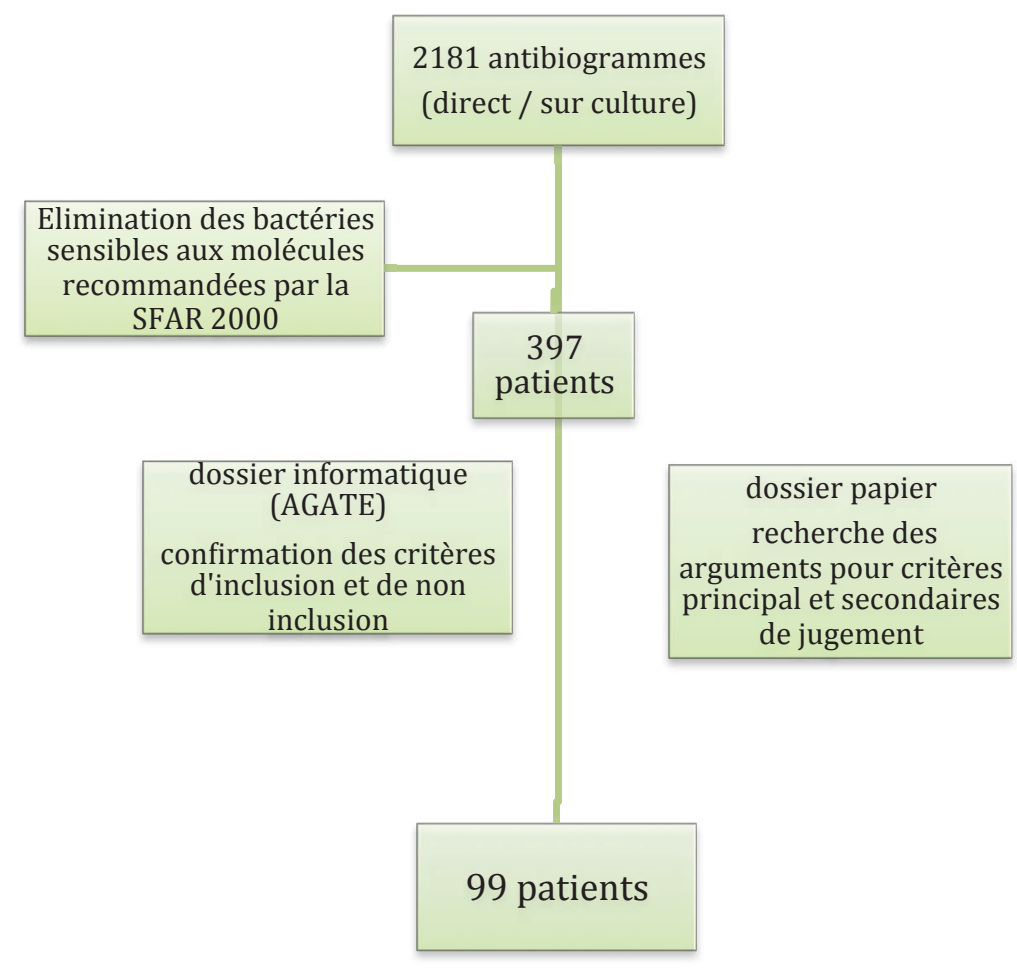

d. Définitions

Le sepsis sévère était défini par une hypotension artérielle (PAS $<100 \mathrm{mmHg}$ ) pour laquelle un remplissage par cristalloide a été prescrit.

Le choc septique était défini par l'introduction d'amines vasopressives pour un soutien hémodynamique.

L'immunodépression était définie par la présence d'un traitement immunosuppresseur ou une corticothérapie supérieure à $10 \mathrm{mg}$ d'hydrocortisone pendant plus 5 jours.

La dénutrition est défini par un score composite :

$\checkmark$ IMC $<18,5$ ou $<21$ si âge $>70$ ans,

$\checkmark$ Pré albuminémie $<0,02 \mathrm{~g} / 1$ dans les 7 jours suivants l'admission. 
L’item «maladie athéromateuse compliquée » comprenait un antécédent de syndrome coronarien aigu, d'accident vasculaire cérébral ischémique, d'artériopathie oblitérante des membres inférieurs, d'anévrisme de l'aorte.

La leucopénie était définie par un taux de globules blancs inférieur à $4.10^{\wedge} 3 / \mathrm{mm}^{\wedge} 3$.

\section{e. $\quad$ Constitution des groupes}

Afin de comparer l'impact d'une antibiothérapie empirique efficace. Nous avons constitué deux groupes,

- le groupe « ATBproba + », correspondant aux péritonites ayant reçu une antibiothérapie probabiliste efficace,

- le groupe «ATBproba - », correspondant aux péritonites dont l'antibiothérapie empirique étaient insuffisante.

\section{Critères de jugement}

Le critère de jugement principal était la mortalité dans les 90 jours des patients présentant une péritonite aiguë communautaire.

Les critères de jugement secondaire étaient :

$\checkmark$ La morbidité dans les 90 jours définie par la présence

- D’une perforation,

- D'un abcès,

- D’une ischémie,

- D'une fistule,

- D’une éviscération/éventration,

- D'une reprise chirurgicale,

- D’un drainage sous contrôle radiologique,

- D’un syndrome de défaillance multi viscéral,

$\checkmark$ La guérison défini par l'absence de complications dans les 90 jours,

$\checkmark$ La durée d'hospitalisation,

$\checkmark$ Le siège de l'étiologie de la péritonite, 
Le mécanisme de la péritonite,

$\checkmark$ L'incidence des différentes associations d'antibiothérapie probabiliste et leurs sensibilités,

$\checkmark$ L’incidence des bactéries retrouvées à la culture et l'étude de leur sensibilité aux antibiotiques,

$\checkmark$ Le délai d'adaptation de l'antibiothérapie empirique, le cas échéant,

$\checkmark$ Le délai de prise en charge chirurgical,

$\checkmark$ La présence d'éléments fongiques à la culture,

$\checkmark$ Pour les patients admis en réanimation, la mortalité, la morbidité, les durées de ventilation, les durées d'hospitalisation en réanimation et globale, la dose maximale d'amines, le taux maximum de lactates artériels, le recours à l'épuration extra rénale,

$\checkmark$ Dans le sous groupe « péritonite appendiculaire », la morbi mortalité définie comme ci dessus.

\section{Analyse statistique}

Les tests statistiques ont été réalisés avec le logiciel STATA 11.(C) StataCorp LP.)

Les variables qualitatives ont été exprimées en nombre et en pourcentages et les variables quantitatives sous forme de moyennes et écarts-types, ou de médianes et extrêmes.

Pour l'analyse univariée, la comparaison des variables qualitatives a été réalisée par le Test de Chi2 ou un Test Exact de Fisher, la comparaison des variables quantitatives par un test $\mathrm{t}$ de Student.

Les tests étaient considérés comme significatifs pour un $\mathrm{p}<0,05$. 


\section{Résultats}

\section{Critères démographiques}

\section{a. Population générale $(n=99)$}

Quatre vingt dix neufs dossiers ont été sélectionnés.

L'âge moyen était de $63,8 \pm 19,5$ ans avec une prédominance féminine (57\%). Un cinquième de notre population était immunodéprimé. Aucun des patients n'était connu porteur d'une bactérie multirésistante, mais $14 \%$ avaient été hospitalisés dans les 6 mois précédent la pathologie aiguë. (Tableau IV)

\section{Tableau IVV}

Critères démographiques de la population et des groupes

\begin{tabular}{|c|c|c|c|c|c|}
\hline & & $\begin{array}{l}\text { Population } \\
\text { globale } \\
\text { n }=99\end{array}$ & $\begin{array}{c}\text { ATBproba }+ \\
\mathbf{n}=\mathbf{3 3}\end{array}$ & $\begin{array}{c}\text { ATBproba - } \\
n=66\end{array}$ & Valeur p \\
\hline \multirow{2}{*}{$\begin{array}{c}\text { Critères } \\
\text { démographiques }\end{array}$} & Age (an) & $63,8 \pm 19,5$ & $64,1 \pm 18,4$ & $63,1 \pm 21,7$ & 0,8 \\
\hline & Sexe (\% Homme) & $43(43)$ & $26(39)$ & $17(52)$ & 0,28 \\
\hline \multirow[t]{10}{*}{ Terrain } & Denutrition $n(\%)$ & $35(35)$ & $22(33)$ & $13(39)$ & 0,3 \\
\hline & Diabète $n(\%)$ & $12(12)$ & $6(9)$ & $6(18)$ & 0,2 \\
\hline & $\begin{array}{l}\text { Insuffisance rénale } \\
\text { chronique } n(\%)\end{array}$ & $6(6)$ & $4(6)$ & $2(6)$ & 1 \\
\hline & Leucopénie $n(\%)$ & $1(1)$ & $1(2)$ & $0(0)$ & 1 \\
\hline & Cancer $n(\%)$ & $18(18)$ & $11(17)$ & $7(21)$ & 0,59 \\
\hline & $\begin{array}{l}\text { Immunodépression } \\
n(\%)\end{array}$ & $20(20)$ & $13(20)$ & $7(21)$ & 1 \\
\hline & $\begin{array}{l}\text { Maladie } \\
\text { athéromateuse } \\
\text { compliquée } n(\%)\end{array}$ & $10(10)$ & 7 (12) & $3(9)$ & Ns \\
\hline & $\begin{array}{l}\text { ATCD } \\
\text { hospitalisation dans } \\
\text { les } 6 \text { mois } n(\%)\end{array}$ & $14(14)$ & $10(15)$ & $4(12)$ & 0,77 \\
\hline & $\begin{array}{l}\text { Antibiothérapie } \\
\text { préalable dans le } \\
\text { mois précédent } n \\
(\%)\end{array}$ & $5(5)$ & $4(6)$ & $1(3)$ & 0,66 \\
\hline & ATCD BMR $n(\%)$ & $0(0)$ & $0(0)$ & $0(0)$ & $\mathrm{Na}$ \\
\hline
\end{tabular}




\section{b. Description des péritonites}

Le mécanisme principal était la perforation digestive dont le siège était majoritairement le colon (76\%). Notons que l'appendicite représentait la majeure partie des perforations coliques.

Un sepsis sévère ou un état de choc septique pré opératoires était retrouvés pour $23 \%$ des patients, sensiblement équivalent au nombre de patient admis en réanimation (24\%). Le taux de mortalité globale s'élevait à 15\%. (Tableau V)

Tableau V

Description des péritonites globales et par groupe

\begin{tabular}{|c|c|c|c|c|c|}
\hline & & $\begin{array}{c}\text { Population } \\
\text { globale } \\
\mathbf{N}=99\end{array}$ & $\begin{array}{l}\text { ATBproba }+ \\
\quad \mathbf{N}=\mathbf{3 3}\end{array}$ & $\begin{array}{l}\text { ATBproba - } \\
\qquad N=66\end{array}$ & Valeur p \\
\hline \multirow[t]{3}{*}{$\begin{array}{l}\text { Mécanisme } \\
\text { n (\%) }\end{array}$} & & & & & $\mathrm{ns}$ \\
\hline & Perforation & $88(89)$ & $59(90)$ & $29(88)$ & \\
\hline & Autre & $13(13)$ & $8(13)$ & $5(15)$ & \\
\hline \multirow{7}{*}{$\begin{array}{l}\text { Siège de la } \\
\text { perforation } \\
\text { n }(\%)\end{array}$} & & & & & $\mathrm{ns}$ \\
\hline & Bilaire & $5(5)$ & $3(5)$ & $2(6)$ & \\
\hline & Gastrique & $3(3)$ & $3(5)$ & $0(0)$ & \\
\hline & Duodénal & $2(2)$ & $2(3)$ & $0(0)$ & \\
\hline & Ileo jéjunal & $14(14)$ & $9(14)$ & $5(15)$ & \\
\hline & Colique & $75(76)$ & 49 (74) & $26(79)$ & \\
\hline & Dont appendice & $39(39)$ & $24(36)$ & $15(45)$ & \\
\hline \multirow[t]{4}{*}{ Gravité } & Sepsis sévère $n(\%)$ & $18(18)$ & $3(9)$ & $15(23)$ & 0,16 \\
\hline & $\begin{array}{l}\text { Choc septique } \\
n(\%)\end{array}$ & $5(5)$ & $2(6)$ & $3(5)$ & 1 \\
\hline & $\begin{array}{l}\text { Lactate pré op } \\
(\mathrm{mmol} / \mathrm{l})\end{array}$ & $2,841,7$ & $2,75 \pm 1,53$ & $2,87 \pm 1,77$ & $\mathrm{~ns}$ \\
\hline & $\begin{array}{l}\text { Hospitalisation en } \\
\text { réanimation }(\mathrm{j})\end{array}$ & $24(24)$ & $7(21)$ & $17(25)$ & 0,62 \\
\hline \multirow{2}{*}{$\begin{array}{l}\text { Délai } \\
\text { thérapeutique } \\
\text { n (\%) }\end{array}$} & $\begin{array}{l}\text { Antibiothérapie } \\
\text { probabiliste }<6 \mathrm{~h}\end{array}$ & $49(50)$ & $13(26,3)$ & $36(73,5)$ & 0,13 \\
\hline & Chirurgie $<6 \mathrm{~h}$ & $38(38)$ & $13(34)$ & $25(65,6)$ & 0,88 \\
\hline
\end{tabular}




\section{Analyse comparative des groupes «ATBproba + » et «ATBproba - »}

\section{a. Données générales}

Sur la période étudiée, 33 patients étaient dans le groupe « ATBproba + » et 66 dans le groupe « ATBproba - ».

Les groupes avaient des critères démographiques généraux comparables (Tableau IV).

\section{b. Péritonites}

Le groupe « ATBproba + » et le groupe «ATBproba - » ne présentaient pas de différence significative quant aux caractéristiques de leurs péritonites. (Tableau V)

Le mécanisme principal de la péritonite était la perforation ( $88 \%$ versus $90 \%)$ au niveau colique, $79 \%$ et $74 \%$, respectivement groupe « ATBproba $+»$ et groupe « ATBproba - ».

Les péritonites d'origine appendiculaire étaient aussi fréquentes dans les deux groupes.

Le groupe «ATBproba - » avait un taux de sepsis sévère pré opératoire plus élevé mais les taux de lactates artériels pré opératoires restaient comparables.

\section{c. Devenir du patient présentant une antibiothérapie probabiliste inadaptée}

1) Mortalité

La mortalité dans le groupe « ATBproba - » était plus élevée mais cette différence n’était pas significative. (Tableau VI)

Tableau V Mortalité hospitalière des patients en cas d'antibiothérapie probabiliste non adaptée

\begin{tabular}{|lcccc|}
\hline & $\begin{array}{c}\text { Population } \\
\text { globale } \\
\mathbf{N}=99\end{array}$ & $\begin{array}{c}\text { ATBproba } \\
\mathbf{N}=33\end{array}$ & $\begin{array}{c}\text { ATBproba - } \\
\mathbf{N}=66\end{array}$ & Valeur p \\
\hline Mortalité $n(\%)$ & $15(15)$ & $4(12,1)$ & $11(16,7)$ & 0,77 \\
\hline
\end{tabular}




\section{2) Morbidité}

Davantage de patients obtenaient une guérison dans les 90 jours en cas d'antibiothérapie probabiliste adaptée $(69,7 \%$ versus $54,5 \%)$. Mais cette différence n'était pas retrouvée significative. (Tableau VII)

De même, les complications post opératoires (fistule, abcès, perforation, ischémie, eviscération) étaient globalement plus fréquentes dans le groupe « ATBproba - » sans qu'aucun résultat ne soit significatif. De même, aucune différence entre les 2 groupes n'existait quand à la reprise chirurgicale ou la réalisation de drainage sous contrôle radiologique.

Sur un plan plus médical, une antibiothérapie probabiliste non adaptée générait plus d'hospitalisation en réanimation. Les patients présentaient d'avantage de syndrome de défaillance multiviscérale en cas d'antibiothérapie initiale inefficace, mais ces différences n'étaient pas non plus significatives.

Enfin, la durée d'hospitalisation semblait plus allongée en cas d'inefficacité de l'antibiothérapie initiale sans que cette différence ne soit significative.

Tableau VIII Morbidité des patients en cas d'antibiothérapie probabiliste inadaptée

\begin{tabular}{|lc|ccc|}
\hline & $\begin{array}{c}\text { Population } \\
\text { globale } \\
\mathbf{N}=99\end{array}$ & $\begin{array}{c}\text { A TBproba } \\
\mathbf{N}=33\end{array}$ & $\begin{array}{c}\text { ATBproba - } \\
\mathbf{N}=66\end{array}$ & Valeur p \\
\hline Guérison $n(\%)$ & $59(60)$ & $23(69,7)$ & $36(54,5)$ & 0,19 \\
\hline Fistule $n(\%)$ & $5(5)$ & $1(3)$ & $4(6)$ & 0,66 \\
\hline Abces $n(\%)$ & $16(16)$ & $4(12,2)$ & $12(18,2)$ & 0,57 \\
\hline Ischémie $n(\%)$ & $3(3)$ & $1(3)$ & $2(3)$ & 1 \\
\hline Perforation $n(\%)$ & $2(2)$ & $0(0)$ & $2(3)$ & 0,55 \\
\hline Eviscération $n(\%)$ & $1(1)$ & $0(0)$ & $1(1,5)$ & 1 \\
\hline Reprise chirurgicale $n(\%)$ & $17(17)$ & $6(18,2)$ & $11(16,7)$ & 1 \\
\hline Drainage per cutané $n(\%)$ & $6(6)$ & $1(3)$ & $5(7,6)$ & 0,66 \\
\hline Hospitalisation en réanimation $n(\%)$ & $24(24)$ & $7(21,2)$ & $17(25,8)$ & 0,8 \\
\hline $\begin{array}{l}\text { Syndrome de défaillance multi } \\
\text { viscéral } n(\%)\end{array}$ & $20(20)$ & $4(12,1)$ & $16(24,2)$ & 0,19 \\
\hline Durée de l'hospitalisation $(j)$ & $21,3 \pm 30,7$ & $17 \pm 21,2$ & $23,5 \pm 34,4$ & 0,32 \\
\hline
\end{tabular}




\section{Aspects microbiologiques}

A. Impact de l'examen direct

L'examen direct était rendu pour $100 \%$ des liquides péritonéaux analysés. Celui ci était compatible avec la culture dans $77 \%$ des cas. Néanmoins, $25,6 \%$ des réponses « flore abondante et variée ». (Figure 2)

Fig. 2. Corrélation entre les résultats de l'examen direct et ceux de la culture.

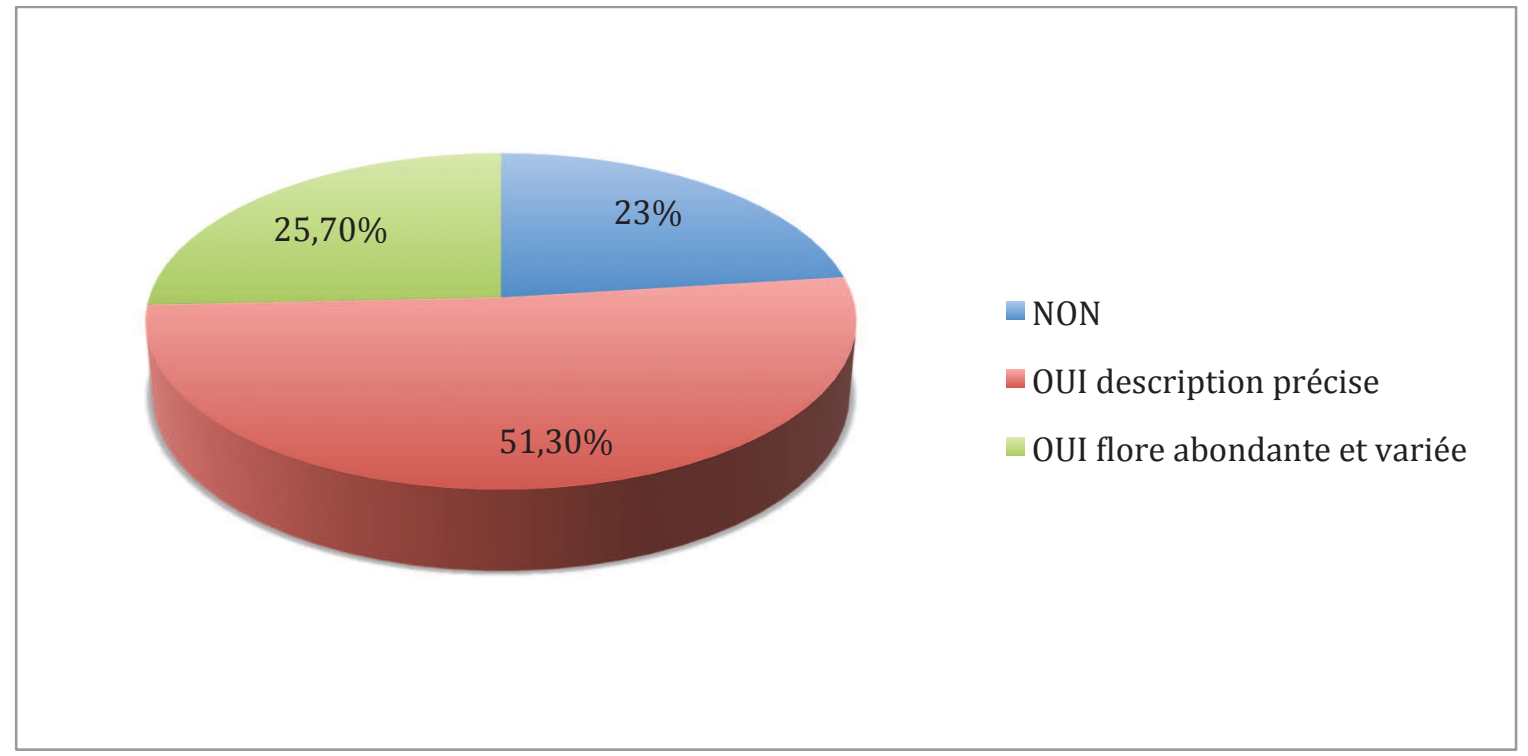

B. Incidence des bactéries

L'étude des 99 dossiers bactériologiques mettait en évidence 291 bactéries.

Chaque culture de liquide péritoneal comportait 2,9 bactéries.

Le détail des espèces bactériennes est représenté par le tableau VIII.

Les entérobactéries étaient les bactéries les plus fréquemment isolées (51\%), avec E.coli en tête $(29 \%)$ et les KES (13\%).

Les cocci Gram positifs (streptocoques et entérocoques) représentaient 29\% de notre panel, suivi par les anaérobies (15\%). 
Notons que $8 \%$ des bactéries appartenaient la famille des bacilles Gram négatif aérobies non fermentant, composée principalement de Pseudomonas sp.

Tableau VIIIII Incidence des bactéries

\begin{tabular}{|c|c|c|}
\hline & \multicolumn{2}{|c|}{$\begin{array}{l}\text { Espèces bactériennes } \\
\qquad(\mathrm{n}=291)\end{array}$} \\
\hline & n & $\%$ \\
\hline Cocci gram positif & 75 & 26 \\
\hline Staphylocoque $s p$ & 3 & 1 \\
\hline Streptocoque sp & 24 & 8 \\
\hline Entérocoque sp & 48 & 16 \\
\hline Entérobactéries & 148 & 51 \\
\hline Escherichia coli & 85 & 29 \\
\hline Proteus sp & 5 & 2 \\
\hline$K E S$ & 39 & 13 \\
\hline Morganella morganii & 2 & 1 \\
\hline Klebsielle $s p$ & 9 & 3 \\
\hline Serratia $s p$ & 1 & $<1$ \\
\hline Enterobacter $s p$ & 10 & 3 \\
\hline Providencia $s p$ & 1 & $<1$ \\
\hline Citrobacter $s p$ & 5 & 2 \\
\hline Hafnia alvei & 7 & 2 \\
\hline Groupe KES non identifié & 4 & 1 \\
\hline Enterobactérie non identifiée & 19 & 7 \\
\hline Bacilles gram négatif non fermentants & 24 & 8 \\
\hline Pseudomonas aeruginosa & 11 & 4 \\
\hline Pseudomonas $s p$ & 12 & 4 \\
\hline Aeromonas hydrophila & 1 & $<1$ \\
\hline Bactéries anaérobies & 44 & 15 \\
\hline Bacteroïdes $s p$ & 10 & 3 \\
\hline Clostridum Perfringens & 3 & 1 \\
\hline Lactobacillus & 2 & $<1$ \\
\hline Autres anaérobies & 29 & 10 \\
\hline
\end{tabular}

C. Profil de sensibilité des bactéries

Sur l'ensemble des dossiers bactériologiques, 183 antibiogrammes ont été analysés. Parmi les bactéries isolées à la culture, un antibiogramme a été fait dans $63 \%$ des cas.

Les antibiogrammes directs, le plus souvent réalisés $(n=61)$ mettaient en évidence une sensibilité des germes à l'Amox/ac.clav de $11 \%$, passant à $64 \%$ pour les $\mathrm{C} 3 \mathrm{G}$ et $62 \%$ pour 
l'Ertapénèm. Les antibiotiques au spectre plus large gardaient une sensiblité plus élevée avec $84 \%$ pour la Tazocilline et $82 \%$ la Ceftazidime.

A l'analyse des antibiogrammes complets $(n=122)$, E.coli avait seulement $35 \%$ de sensibilité à l'Augmentin. Pour les autres antibiotiques cette sensibilité s'élèvait à plus de 79\%.

10 entérobactéries étaient productrice de BLSE. Il n’existait aucune carbapénémase.

Les cocci Gram positif, les bacilles gram négatifs non fermentants et les anaérobies gardaient une sensibilité élevée à l'ensemble des antibiotiques testés, en dehors de leur résistance naturelle. (Tableau IX)

Tableau VIIIX Profil de sensibilité des bactéries

\begin{tabular}{|c|c|c|c|c|c|c|c|c|c|c|c|}
\hline & 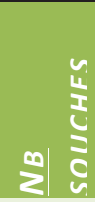 & 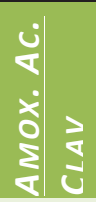 & \begin{tabular}{l|l}
$\frac{5}{x}$ & \\
$\frac{1}{5}$ & \\
0 & \\
4 & \\
4 & 4
\end{tabular} & 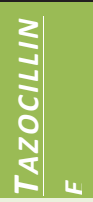 & 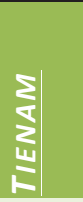 & 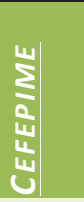 & $\frac{3}{\frac{3}{N}}$ & $\frac{\bar{u}}{2}$ & $\begin{array}{l}3 \\
\vdots \\
z \\
\frac{1}{4}\end{array}$ & $\begin{array}{l}x \\
0 \\
4 \\
u\end{array}$ & 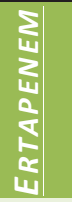 \\
\hline & 183 & S & $\mathrm{S}$ & $\mathrm{S}$ & $\mathrm{S}$ & $\mathrm{S}$ & S & S & S & $\mathrm{S}$ & $\mathrm{S}$ \\
\hline Antibiogramme Direct & 61 & 11 & 64 & 84 & - & - & 82 & - & - & - & 62 \\
\hline \multicolumn{12}{|l|}{ Cocci Gram + } \\
\hline Staphylocoque sp & 1 & 100 & - & - & - & - & - & - & - & - & - \\
\hline Streptocoque sp & 3 & 100 & - & - & - & - & - & - & 100 & - & - \\
\hline enterocoque sp & 13 & 100 & - & - & - & - & - & - & 40 & - & - \\
\hline Faecalis & 2 & 100 & - & - & - & - & - & - & - & - & - \\
\hline Faecium & 2 & 50 & - & - & - & - & - & - & 0 & - & - \\
\hline \multicolumn{12}{|l|}{ Bacille Gram - } \\
\hline E.Coli & 52 & 35 & 82 & 79 & 100 & 83 & 80 & 94 & 100 & 85 & 100 \\
\hline Proteus sp & 2 & 100 & 100 & 100 & 100 & 100 & 100 & 0 & 100 & 100 & 100 \\
\hline Morganella morganii & 1 & 0 & 100 & 100 & 100 & 100 & 100 & 100 & 100 & 100 & 100 \\
\hline Klebsielle sp & 6 & 100 & 100 & 100 & 100 & 100 & 100 & 100 & 100 & 100 & 100 \\
\hline Serratia sp & 1 & 0 & 100 & 100 & 100 & 100 & 100 & 0 & 0 & 100 & 100 \\
\hline Enterobacter sp & 7 & 0 & 71 & 71 & 100 & 83 & 66 & 100 & 100 & 100 & 83 \\
\hline Citrobacter sp & 2 & 0 & 100 & 50 & 100 & 80 & 50 & 100 & 100 & 100 & 100 \\
\hline Hafnia alvei & 6 & 0 & 83 & 83 & 100 & 100 & 100 & 100 & 100 & 100 & 100 \\
\hline Providencia sp & 1 & 0 & 0 & 0 & 100 & 100 & 0 & 0 & 0 & 100 & - \\
\hline Enterobacterie non identifiée & 1 & 100 & - & 100 & 100 & - & - & - & 0 & - & - \\
\hline \multicolumn{12}{|l|}{ Bacilles Gram négatif non fermentants } \\
\hline Pseudomonas aeruginosa & 8 & - & - & 100 & 100 & 100 & 100 & - & 100 & 100 & - \\
\hline Pseudomonasp & 1 & - & - & 100 & 100 & 100 & 100 & - & 100 & 100 & - \\
\hline Aeromonas hydrophyla & 1 & 0 & 100 & 100 & 0 & 100 & 100 & 100 & - & 100 & 0 \\
\hline Anaérobie & 12 & 92 & 100 & 92 & 100 & 100 & 100 & - & - & 100 & - \\
\hline
\end{tabular}




\section{Evaluation fongique}

Parmi les 99 liquides péritonéaux étudiés, 4 (4\%) présentaient une culture fongique positive à Candida sp. Tous les patients ont été traités.

\section{Aspects thérapeutiques}

A. Antibiothérapie probabiliste prescrite

i. Sensibilité des antibiothérapies délivrées

Au total, 17 schémas thérapeutiques ont été recensés. La figure 3 résume l'efficacité théorique des antibiotiques probabilistes au vu des résultats de chaque patient.

L'association aminoside - Amox/ac.clav avait une efficacité de seulement 21\%, l'association C3G - Métronidazole 46,6\%. L'association Tazocilline- aminoside avait une efficacité de $60 \%$.

Seules 5 des 17 shémas d'antibiothérapies (administrés chez 11 patients au total) avaient une efficacité supérieure à $50 \%$.

\section{Fig. 3. Antibiothérapie probabiliste et efficacité}

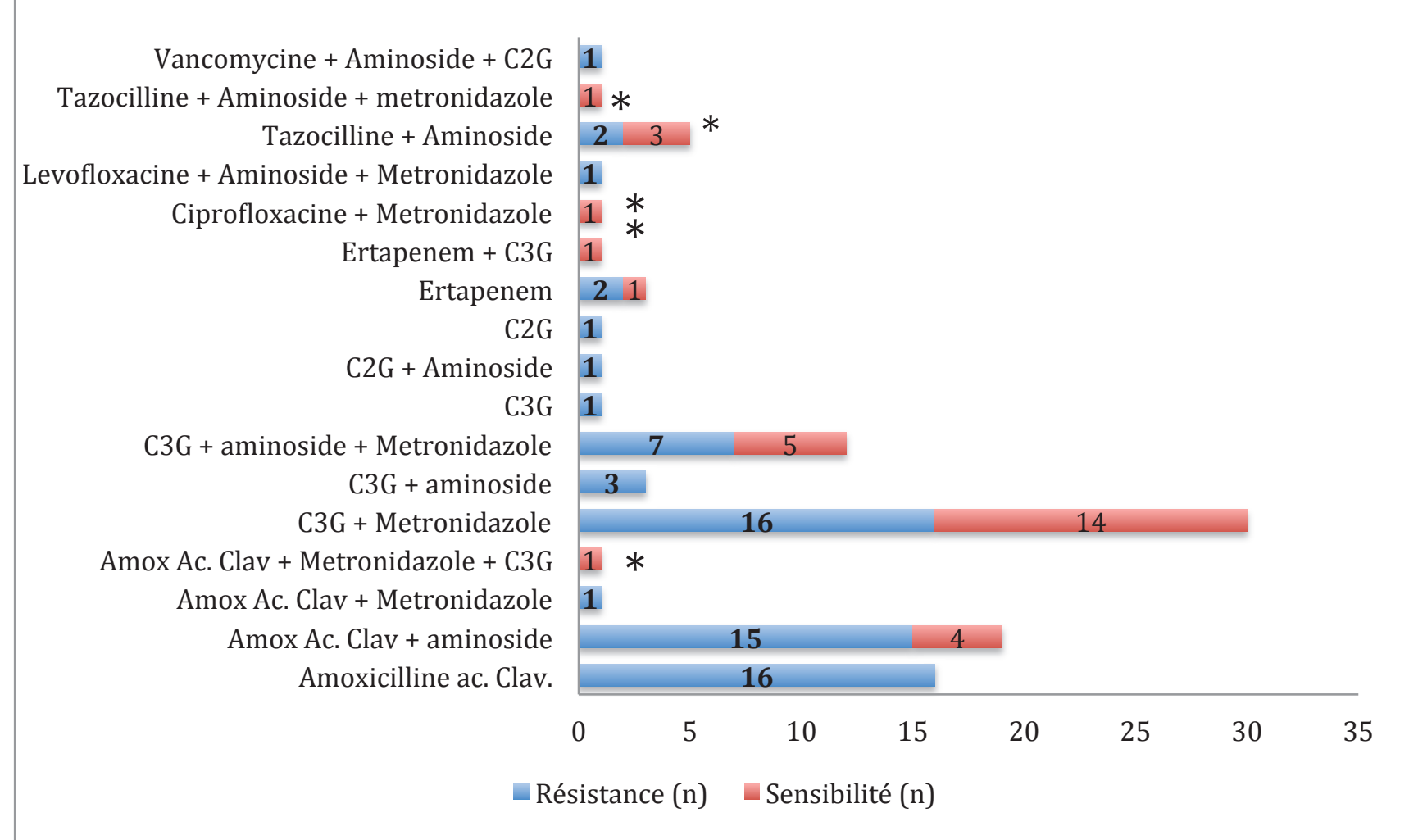


ii. Conformité de l'antibiothérapie probabiliste au consensus

Seuls 55 patients ont reçu un schéma thérapeutiques correspondant à ceux proposés par la Conférence de Consensus de 2000. (figure 4)

Fig.4. Pourcentage d'antibiothérapie probabiliste administrée recommandée par la Conférence de Consensus SFAR 2000.

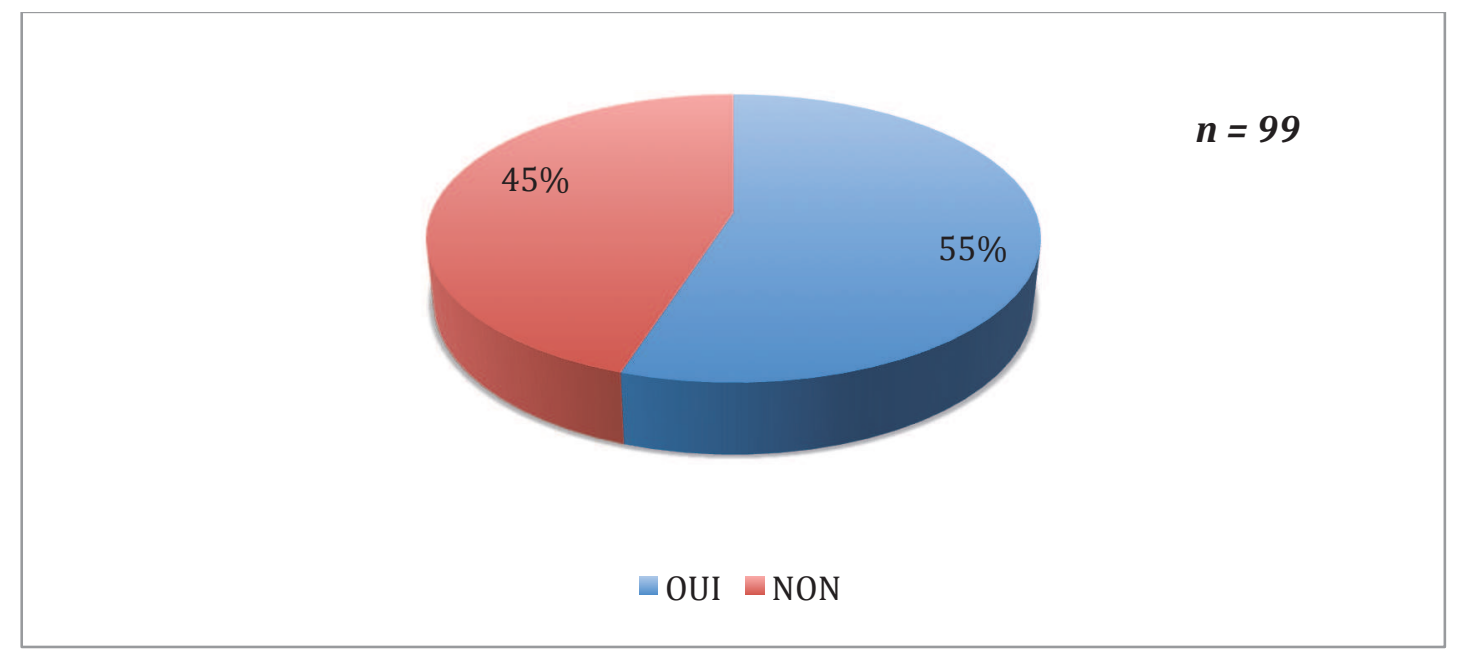

iii. Efficacité des différentes antibiothérapies proposées par le consensus

Le tableau X résume l'efficacité théorique des différentes antibiothérapies probabilistes du consensus français.

Sur notre échantillon de 99 péritonites, l'association Amox/Ac.clav. - Gentamycine aurait été efficace chez 61 patients (62\%), sachant que l'Amox/Ac.clav seul aurait été eficace pour seulement $47 \%$ des patients. (Tableau X)

L'association C3G - Métronidazole aurait été active pour 39 péritonites (39\%).

Enfin la Tazocilline aurait été efficace dans $82 \%$ des cas.

A noter que $100 \%$ des péritonites auraient été traitées efficacement par l'association Aminoside-Metronidazole. (schéma d'antibiotiques non prescrites dans notre étude) 
Tableau X Nombre de péritonites sensibles à l'antibiothérapie consensuelle.

\begin{tabular}{|lc|}
\hline \multicolumn{1}{|c|}{ Augmentin seul } & $\begin{array}{c}\text { Sensible } \\
\mathbf{N}=99 \\
\text { n }(\%)\end{array}$ \\
\hline Augmentin + Gentamycine & $61(62)$ \\
\hline Claventin + Gentamycine & $47(47)$ \\
\hline \multicolumn{1}{|c|}{ Claventin seul } & $89(90)$ \\
\hline Céphalosporine 3G + Métronidazole & $75(76)$ \\
\hline Cefoxitine & $39(39)$ \\
\hline Aminoside + Metronidazole & $39(39)$ \\
\hline Cefepime + Metronidazole & $99(100)$ \\
\hline Tazocilline & $60(61)$ \\
\hline
\end{tabular}

iv. Délai moyen d'instauration de l'antibiothérapie

Dans la moitié des cas (49\%), l'antibiothérapie probabiliste était administrée dans les $6 \mathrm{~h}$ suivant le diagnostic.

A l'inverse, celle ci était injectée au moins $12 \mathrm{~h}$ après le diagnostic dans $37 \%$ des dossiers. (Figurre 5)

Fig.5. Délai d'instauration de l'antibiothérapie probabiliste (pourcentage)

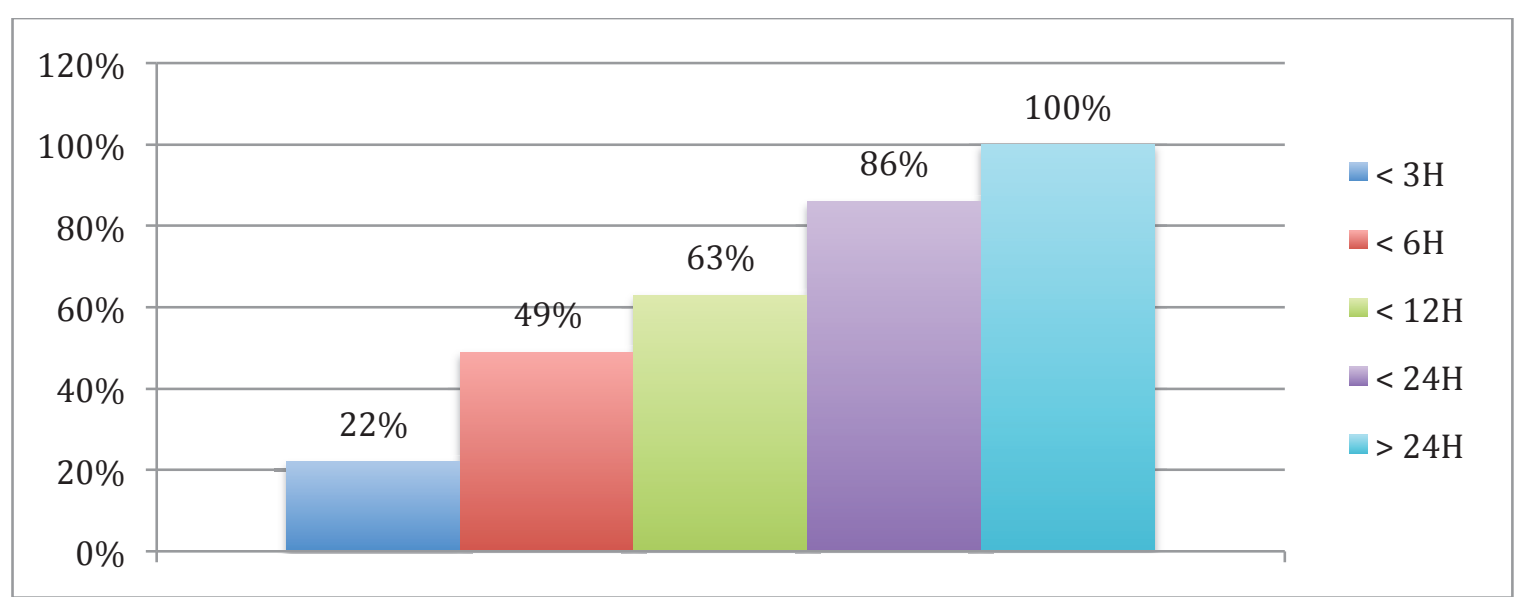


Les patients présentant un état de sepsis sévère ou choc septique en pré opératoire avaient un délai médian d'instauration d'une antibiothérapie probabiliste de $3 \mathrm{~h}$.

\section{B. Délai d'instauration d'une antibiothérapie efficace}

La répartition du délai d'instauration d'une antbiothérapie efficace est représentée sur la figure 6. Un quart des patients avaient reçu une thérapeutique adaptée dans les $24 \mathrm{~h}$, un quart dans les 3 jours.

Cependant, un quart des patients avaient eu leur antibiothérapie réajustée 4 jours après le diagnostic.

Un point intéressant est que plus de $20 \%$ des patients n'avaient pas eu réadaptation de l'antibiothérapie sur les germes retrouvés.

Dans le groupe «ATBproba - », 80\% des patients ont finalement reçu un traitement efficace après adaptation aux résultats bactériologiques, tandis que $20 \%$ sont restés avec une antibiothérapie inefficace vis à vis de l'ensemble des germes retrouvés. (figure 7)

Fig. 6. Délai d'instauration d'une antibiothérapie efficace (pourcentage)

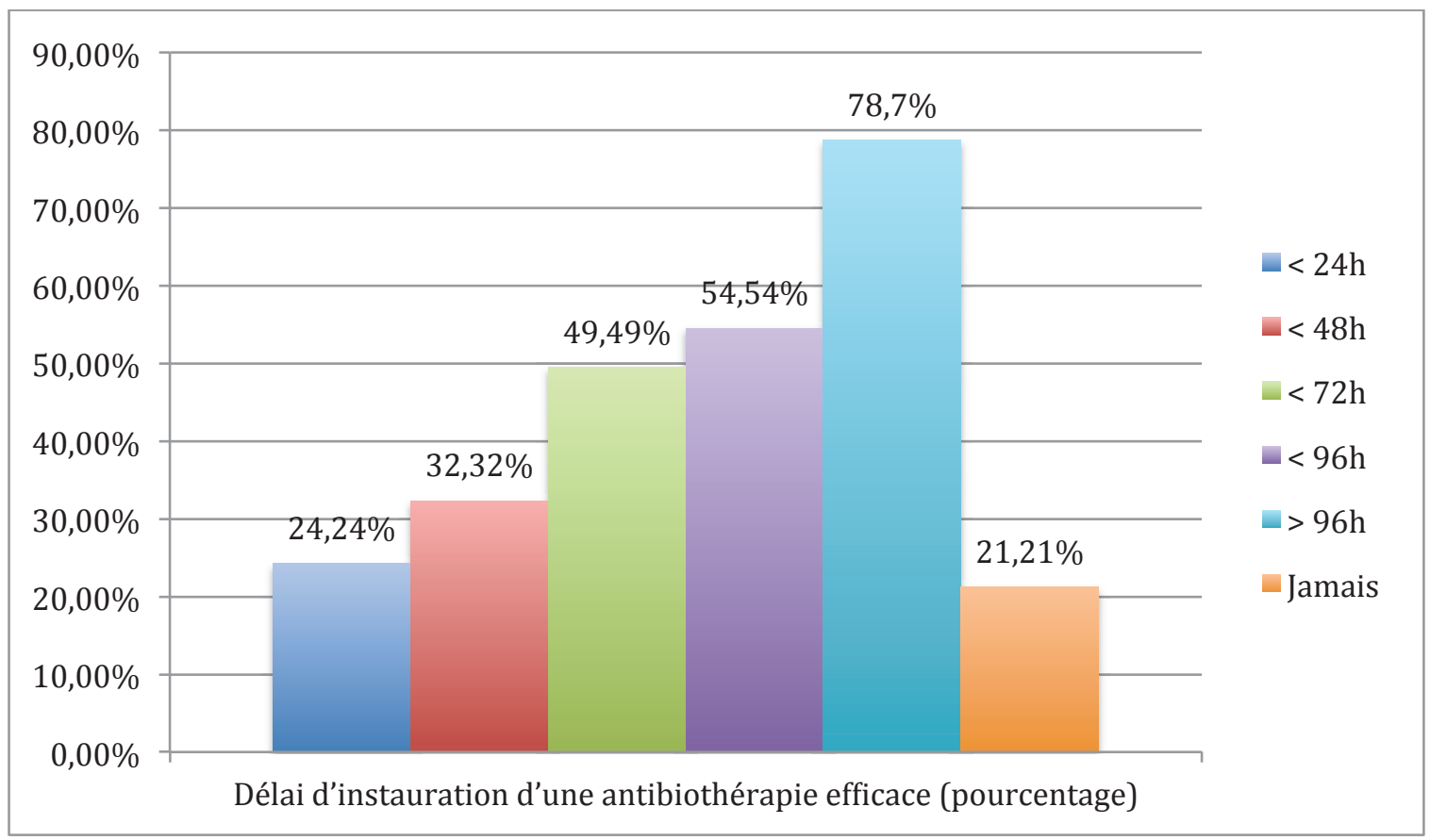


Fig. 7. Antibiothérapie réadaptée au sein du groupe «ATBproba - » $(n=66)$

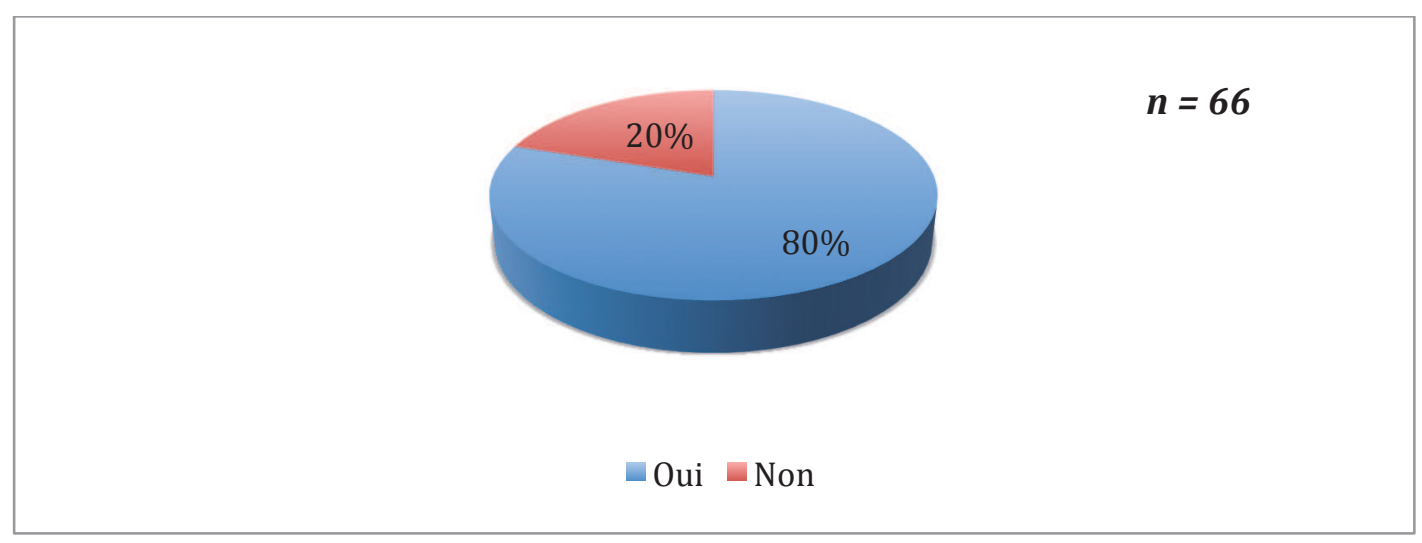

C. Délai de prise en charge chirurgicale

Une fois le diagnostic posé, le patient était opéré dans la moitié des cas au plus tard dans les $12 \mathrm{~h}$. Plus de $75 \%$ des patients avaient été opérés dans les 24 premières heures. Seulement 13\% bénéficiaient d'un traitement chirurgical dans les 3h suivant l'imagerie. (Figure 8)

En cas de sepsis sévère ou de choc septique, le delai médian de prise en charge chirurgicale était de $3 \mathrm{~h} 30$.

Fig.8. Délai de prise en charge chirurgicale (pourcentage)

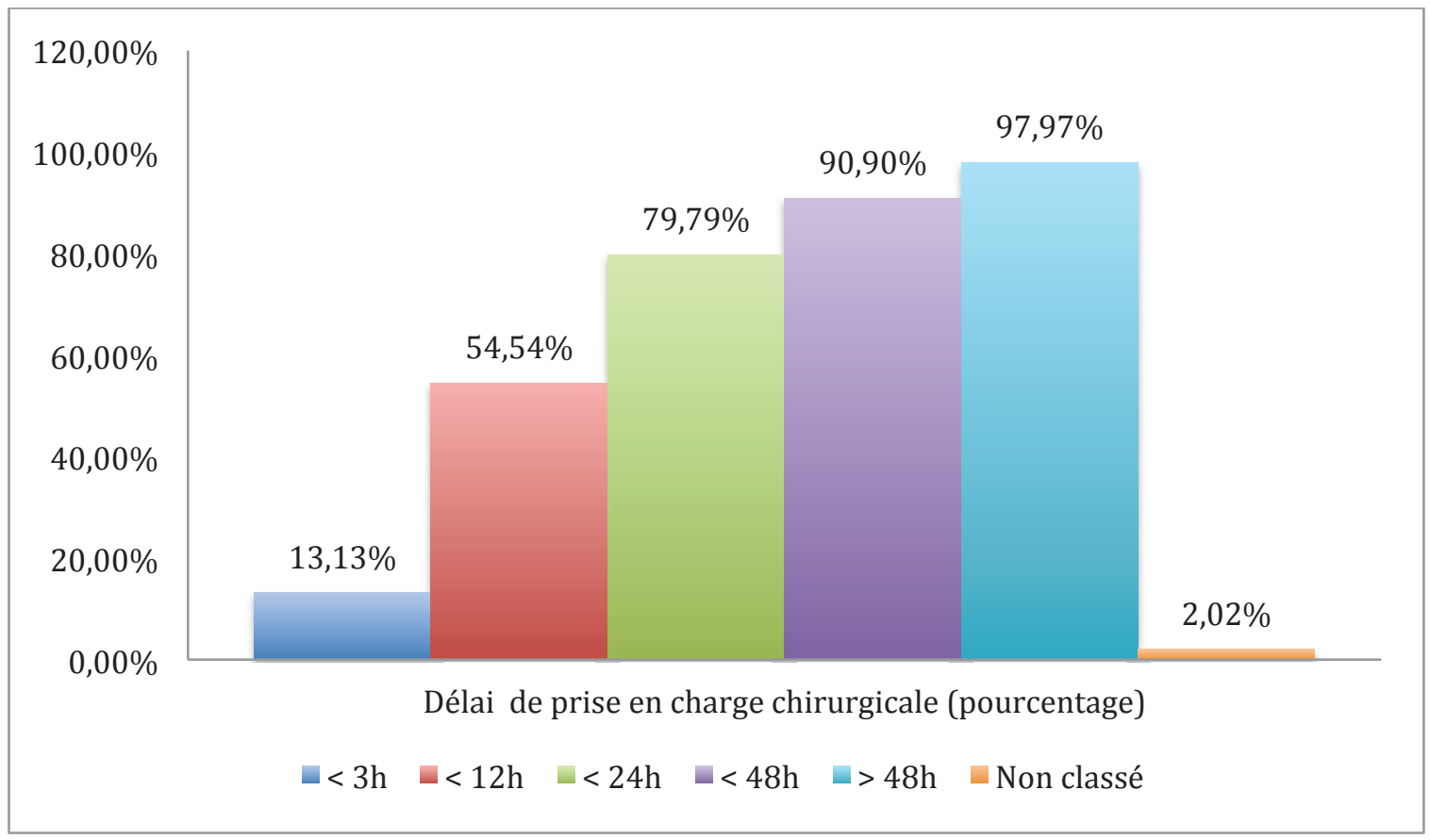




\section{Etude du sous-groupe : « réanimation »}

Vingt quatre (24) patients des 99 étudiés ont été admis en réanimation. L'IGS2 médian à l'admission était de 54 [25-96]. L'âge médian était de 73 [42-90] ans. Cinq patients étaient en état de choc pré opératoire, 13 en sepsis sévère.

La durée médiane de ventilation était de 14 [0-132] jours. La durée médiane de séjour en réanimation était de 15 [2-65] jours. Le taux de mortalité en réanimation était de $46 \%, 54 \%$ en hospitalier.

Les critères démographiques, les caractéristiques de la péritonite et les comorbidités étaient comparable entre les deux groupes. (Tableau XI)

Les patients étaient répartis en 2 groupes, l'un ayant reçu une antibiothérapie probabiliste active «ATBproba + » $(\mathrm{n}=7)$ et l'autre une antibiothérapie probabiliste inactive «ATBproba-» $(\mathrm{n}=17)$. L'analyse comparative des 2 échantillons ne mettait en évidence qu'une seule différence significative concernant le taux de sepsis sévère pré opératoire. (Tableau XII)

Tableau IX

Critères démographiques des patients admis en réanimation

\begin{tabular}{|c|c|c|c|c|c|}
\hline & & $\begin{array}{l}\begin{array}{c}\text { Population } \\
\text { globale } \\
\mathbf{N}=\mathbf{2 4}\end{array} \\
\end{array}$ & $\begin{array}{c}\text { Groupe } \\
\text { «ATBproba + » } \\
\mathbf{N}=7\end{array}$ & $\begin{array}{c}\text { Groupe } \\
\text { «ATBproba - » } \\
\mathbf{N}=17\end{array}$ & p \\
\hline \multirow{2}{*}{$\begin{array}{c}\text { Critères } \\
\text { démographiques }\end{array}$} & Age (an) & $73[42-90]$ & 75,9 & 68,6 & 0,18 \\
\hline & Sexe (\% Homme) & $6(25)$ & $2(29)$ & $4(24)$ & \\
\hline \multirow{10}{*}{ Terrain } & Denutrition $n(\%)$ & $24(37)$ & $3(42)$ & $6(35)$ & \\
\hline & Diabète $n(\%)$ & $5(21)$ & $4(57)$ & $1(6)$ & \\
\hline & $\begin{array}{l}\text { Insuffisance rénale } \\
\text { chronique } n(\%)\end{array}$ & $1(4)$ & $0(0)$ & $1(6)$ & \\
\hline & Leucopénie $n(\%)$ & $1(4)$ & $0(0)$ & $1(6)$ & \\
\hline & Cancer $n(\%)$ & $5(21)$ & $2(29)$ & $3(18)$ & \\
\hline & $\begin{array}{l}\text { Immunodépression } \\
n(\%)\end{array}$ & $9(38)$ & $3(43)$ & $6(35)$ & \\
\hline & $\begin{array}{l}\text { Maladie athéromateuse } \\
\text { compliquée } n(\%)\end{array}$ & $4(17)$ & $0(0)$ & $4(24)$ & \\
\hline & $\begin{array}{l}\text { ATCD hospitalisation } \\
\text { dans les } 6 \text { mois } n(\%)\end{array}$ & $4(17)$ & $0(0)$ & $4(24)$ & \\
\hline & $\begin{array}{l}\text { Antibiothérapie } \\
\text { préalable dans le mois } \\
\text { précédent } n(\%)\end{array}$ & $3(13)$ & $1(14)$ & $2(12)$ & \\
\hline & ATCD BMR $n(\%)$ & $0(0)$ & $0(0)$ & $0(0)$ & \\
\hline
\end{tabular}


Tableau XII

Description de la population réanimatoire

\begin{tabular}{|c|c|c|c|c|c|}
\hline & & $\begin{array}{l}\text { Population } \\
\text { globale } \\
\mathbf{N}=\mathbf{2 4} \\
\end{array}$ & $\begin{array}{c}\text { Groupe } \\
\text { «ATBproba + » } \\
\mathbf{N}=7\end{array}$ & $\begin{array}{c}\text { Groupe } \\
\text { «ATBproba - » } \\
\mathbf{N}=\mathbf{1 7}\end{array}$ & $\mathbf{p}$ \\
\hline \multirow{2}{*}{$\begin{array}{c}\text { Mécanisme } \\
n(\%)\end{array}$} & Perforation & $20(83)$ & $5(82)$ & $13(76)$ & \\
\hline & Autre & $6(25)$ & $2(28)$ & $4(24)$ & \\
\hline \multirow{6}{*}{$\begin{array}{l}\text { Siège } \\
n(\%)\end{array}$} & Bilaire & $3(13)$ & $1(14)$ & $2(12)$ & \\
\hline & Gastrique & $1(4)$ & $0(0)$ & $1(6)$ & \\
\hline & Duodénal & $0(0)$ & $0(0)$ & $0(0)$ & \\
\hline & Ileo jéjunal & 7 (29) & $3(43)$ & $4(24)$ & \\
\hline & Colique & $13(54)$ & $3(43)$ & $10(59)$ & \\
\hline & $\begin{array}{l}\text { Dont appendice } n \\
(\%)\end{array}$ & $0(0)$ & $0(0)$ & $0(0)$ & \\
\hline \multirow[t]{4}{*}{ Gravité } & IGS2 & $54[25-96]$ & $48[28-77]$ & $53[25-96]$ & \\
\hline & Sepsis sévère $n(\%)$ & $13(54)$ & $1(14)$ & $12(71)$ & 0,02 \\
\hline & Choc septique $n(\%)$ & $5(21)$ & $2(29)$ & $3(18)$ & \\
\hline & Lactate pré op mmol/l & $4,38[1,7-6,5]$ & $4,640,75-19,3]$ & $6,8[2,5-20,1]$ & \\
\hline $\begin{array}{c}\text { Syndrome de } \\
\text { défaillance multi } \\
\text { viscérale } \\
n(\%)\end{array}$ & & $17(71)$ & $4(57,1)$ & $13(76,5)$ & 0,37 \\
\hline \multirow{3}{*}{$\begin{array}{l}\text { Durée moyenne } \\
\text { (j) }\end{array}$} & Ventilation & $14[0-132]$ & $7,5[2-50]$ & $18[0-132]$ & 0,61 \\
\hline & $\begin{array}{l}\text { Hospitalisation en } \\
\text { réanimation }\end{array}$ & $15,5[2-65]$ & $9,5[3-50]$ & $18[2-65]$ & 0,59 \\
\hline & Hospitalisation & 29 [2-201] & $29[3-111]$ & $36[2-201]$ & 0,47 \\
\hline \multirow[t]{2}{*}{$\begin{array}{c}\text { Mortalité } \\
n(\%)\end{array}$} & En réanimation & $11(46)$ & $4(57,1)$ & $7(41,2)$ & 0,66 \\
\hline & Hospitalière & $13(54)$ & $4(57,1$ & $9(52,9)$ & \\
\hline
\end{tabular}

Dans le sous-groupe «réanimation », la mortalité semblait ne pas être plus élevée en cas d'antibiothérapie inadaptée. Ce résultat était non significatif.

La durée d'hospitalisation en réanimation, la durée de ventilation et la durée de séjour hospitalier étaient plus importantes en cas d'antibiothérapie initiale inefficace. (Résultats non significatifs) 
Les patients présentaient plus souvent de syndrome de défaillance multiviscéral en cas d'antibiothérapie probabiliste résistante.

\section{Etude du sous-groupe « péritonite appendiculaire »}

Dans notre étude, nous avons relevé 39 péritonites d'origine appendiculaire. $61 \%$ d'entre elles n'avaient pas reçu une antibiothérapie probabiliste efficace. (Figure 9)

Fig.9. Pourcentage d'antibiothérapie probabiliste efficace en cas de péritonite appendiculaire.

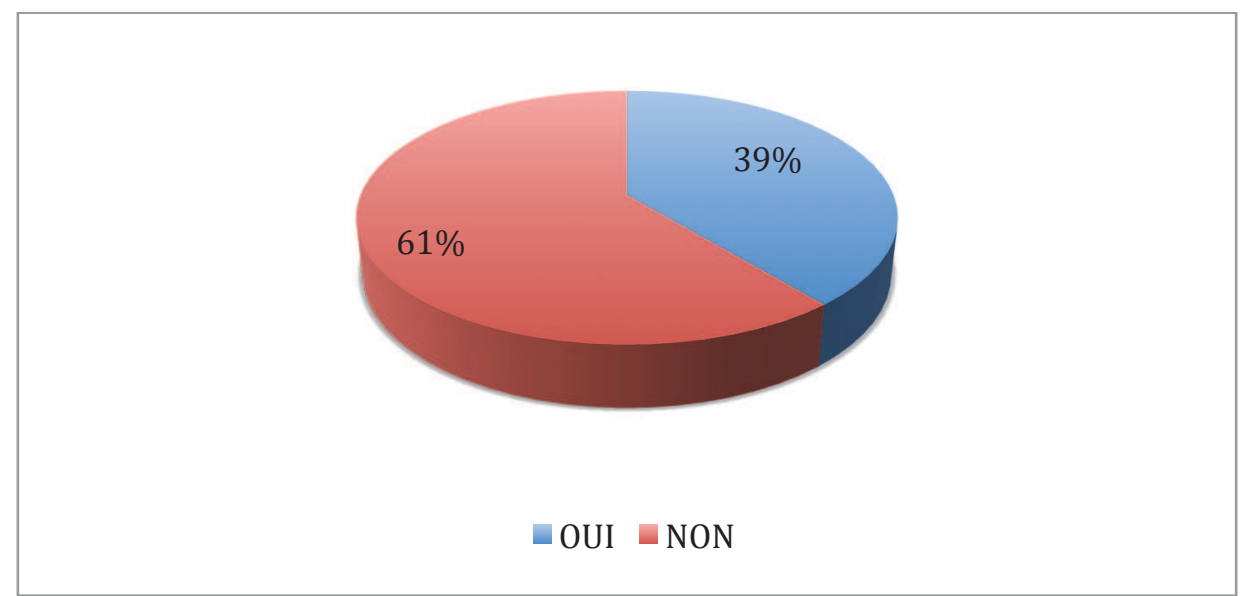

Aucun de ces patients ne décéda ou fut hospitalisé en réanimation.

Ceux qui recevaient une antibiothérapie efficace avaient un taux de guérison sensiblement plus élevé. (non significatif)

Les patients ayant une péritonite appendiculaire présentaient très peu de complications.

Six patients sur 24 n'avaient pas eu leur antibiothérapie réadaptée. Cependant, un seul avait présenté un abcès résiduel. (Tableau XIII) 
Tableau XIII

Guérison et complications en cas de péritonite appendiculaire.

\begin{tabular}{|c|c|c|c|c|}
\hline & $\begin{array}{c}\begin{array}{c}\text { Population } \\
\text { globale } \\
\mathbf{N}=39\end{array} \\
\end{array}$ & $\begin{array}{c}\text { «ATBproba } \\
+» \\
\mathbf{N}=15\end{array}$ & $\begin{array}{c}\text { «ATBproba - } \\
\text { " } \\
\mathbf{N}=\mathbf{2 4}\end{array}$ & p \\
\hline Mortalité & 0 & 0 & 0 & NA \\
\hline Guérison & $33(85)$ & $14(93,3)$ & $19(79,2)$ & 0,38 \\
\hline Fistule & 0 & 0 & 0 & NA \\
\hline Abces & $5(13)$ & $1(6,7)$ & $4(16,7)$ & 0,63 \\
\hline Ischémie & 0 & 0 & 0 & NA \\
\hline Perforation & 0 & 0 & 0 & NA \\
\hline Eviscération & $1(3)$ & $0(0)$ & $1(4,2)$ & 1 \\
\hline Reprise chirurgicale & 0 & 0 & 0 & NA \\
\hline Drainage per cutané & $4(10)$ & $0(0)$ & $4(16,7)$ & 0,15 \\
\hline $\begin{array}{l}\text { Hospitalisation en } \\
\text { réanimation }\end{array}$ & 0 & $0(0)$ & $0(0)$ & NA \\
\hline $\begin{array}{l}\text { Syndrome de } \\
\text { défaillance } \\
\text { multiviscéral }\end{array}$ & 0 & 0 & 0 & NA \\
\hline
\end{tabular}

NA : non applicable 


\section{Discussion}

\section{Devenir}

Tout comme la chirurgie, l'antibiothérapie probabiliste est un des axes majeurs de la prise en charge des péritonites. Notre étude visait à analyser la qualité de la prescription de l'antibiothérapie probabiliste dans le cadre des péritonites communautaires, et notamment l'impact d'un schéma thérapeutique inadapté sur le pronostic en terme de mortalité.

Notre taux de mortalité globale est relativement faible (15\%). La littérature retrouve une mortalité variant de 4 à 45\% [1, 10,11, 13, 14, 39, 55]. Les différents taux de mortalité entre les séries sont difficiles à comparer, du fait de la très grande diversité possible des péritonites. (sévérité, germes en cause, siège, mécanisme, classifications multiples, caractère communautaire ou nosocomial).

Dans notre série, la mortalité, n'était pas significativement différente selon que l'antibiothérapie probabiliste était efficace ou non $(12,1 \%$ vs $16,7 \%, p=0,77)$. Ce résultat principal mérite d'être commenté.

Sur le point précis de l'impact sur la mortalité d'une antibiothérapie probabiliste non adaptée dans le cadre des péritonites, la littérature internationale est discordante. Par exemple, en 1996, Koperna et coll. concluait qu'il existait une sumortalité en cas d'antibiothérapie non adaptée, de même Montavers et coll. et Mosdell et coll. [40, 56, 57]. Mais la méthodologie ne s'intéressait pas uniquement à l'antibiothérapie initiale. A l'inverse, d'autres séries de la littérature plus récentes ne mettait pas en évidence de surmortalité liée à l'inefficacité de l'antibiothérapie initiale $[2,14,58]$. En faisant le parallèle avec les péritonites nosocomiales, qui ont été bien plus étudiées, la surmortalité due à une antibiothérapie probabiliste non adaptée est en revanche bien démontrée, notamment dans les travaux de Mosdell et al, et Montravers et al( ref) .

Ce défaut de significativité de ce résultat peut être expliqué par un manque de puissance dû à un effectif trop faible pour émettre une comparaison sur un évènement peu fréquent (mortalité de $15 \%$ dans notre étude). Les effectifs des autres études traitant la même problématique comportaient au minimum 175 patients [59].

L'effectif plus réduit de notre série est surtout dû à la manière dont nous avons sélectionné nos 
patients. Au CHU de Rouen, le caractère "communautaire" d'une péritonite n'est pas codé, ni en Chirurgie, ni en Bactériologie, ni au Département d'Epidémiologie et de Santé Publique (DESP). C'est la raison pour laquelle nous nous sommes basés au départ sur la liste de tous les prélèvements péritonéaux positifs recencés au laboratoire de Bactériologie. De plus, nous n'avons retenu que les germes des liquides péritonéaux résistants aux antibiothérapies du Consensus Français. En effet, si nous avions inclus l'ensemble des péritonites communautaires, la proportion de bactéries multisensibles aurait été très importante, déséquilibrant énormément l'effectif des deux groupes «ATB proba + » et «ATBproba - », limitant la réalisation des tests comparatifs entre ces groupes. Ce mode de sélection se rapproche au final d'une méthodologie par appariement : il n'existe en effet aucune différence significative entre nos deux groupes en terme d'age, de sexe, de comorbité ou de gravité de la péritonite.

Dans notre travail, l'analyse comparative de la morbidité en fonction de l'efficacité ou non de l'antibiothérapie probabiliste n'a pas montré de différence. En effet, la durée d'hospitalisation semble allongée $(34,4 \mathrm{j})$, comme les syndromes de défaillance multiviscérale (24\%), le taux de guérison moins important (54,5\%), les complications majorées, principalement sous forme d'abcès post opératoire (18,2\%). L'absence de significativité de ces résultats ne nous permet pas de conclure avec certitude. Nos résultats sont en désaccord avec la littérature. Hopskins et coll. en 1993 mettait en évidence qu'une erreur dans l'antibiothérapie empirique était à l'origine de davantage complications post opératoires [59]. De même, Mosdell et coll. a montré, comme Falagas et coll., qu'une antibiothérapie probabiliste inadaptée augmentait la morbidité, les complications chirurgicales et les durées de séjour [60]. Cette augmentation de morbidité est bien expliquée physiologiquement. En effet, un spectre antibactérien insuffisant et/ou le retard d'une prise en charge antibiotique peuvent entrainer la persistance d'un ou plusieurs germes à minima malgré un traitement chirurgical optimal. L'objectif de cette première antibiothérapie est de diminuer la fréquence des abcès résiduels, de traiter correctement une bactériémie et d'en diminuer l'incidence [61].

Dans la littérature, des auteurs ont mis en évidence que certains scores de gravité pouvaient être corrélés à la morbi-mortalité post-opératoire. Il s'agit de scores non spécifiques comme l'APACHE 2 et l'IGS2 ou de scores spécifiques pour les peritonites, le MPI (Mannheim Peritonitis Index) et le PIA (Peritonitis Index Altona) [16, 17, 62-64]. Dans notre travail, nous n'avons pas pu calculer ces scores de sévérité du fait du caractère rétrospectif de notre analyse. De même, la gravité évaluée précisément par le chirurgien aurait été pertinente à 
relever, mais cette information était difficilement analysable par la seule analyse rétrospective des observations et des compte-rendus opératoires. Par exemple le caractère généralisé de la péritonite n'aura pas le même devenir qu'un abcès péri-appendiculaire.

Cependant, il nous paraissait intéressant d'étudier 2 sous-groupes de gravité extrêmement différente : les plus sévères, les péritonites ayant entrainé une hospitalisation en réanimation, et à l'inverse, les moins sévères, les péritonites d'origine appendiculaires.

Dans notre série, $24 \%$ des patients ont été admis en réanimation, avec un IGS2 médian à 54 [25; 96] traduisant bien la sévérité importante de cette infection en réanimation. Cette gravité pouvait être liée soit aux germes (comme les entérobactéries par exemple), soit aux comorbidités des patients, soit à un éventuel retard de prise en charge thérapeutique, qu'il soit d'ordre médical (échec de la première antibiothérapie, délai d'introduction tardif, absence de réadaptation à $72 \mathrm{~h} . .$. ) ou chirurgical (délai de prise en charge prolongée, contrôle chirurgical non optimal...) [39].

Il est intéressant de noter que dans notre étude, la mortalité n'était pas différente selon que l'antibiothérapie initiale soit efficace ou non. Aucune étude n'a été publiée sur cette question précise de l'impact d'une inefficacité de l'antibiothérapie probabiliste dans les péritonites communautaires en réanimation. Par contre, d'autres travaux ont bien démontré qu'il existait un lien entre inefficacité de l'antibiothérapie probabiliste et morbi-mortalité, tous types d'infection confondus [65]. De plus, on rappelle que cet impact sur la morbi-mortalité est démontré sur l'ensemble des péritonites communautaires, graves ou non [40, 56, 57]. L'urgence à l'administration d'une antibiothérapie adaptée (donc sans erreur de schéma probabiliste) est un des grands principes de prise en charge des sepsis sévères ou choc septiques rappelé par la Surviving Sepsis Campaign [66].

Le faible effectif de notre population de réanimation (24 patients) était le facteur principal limitant la puissance de l'étude de cette sous-population. Malgré l'absence de significativité, la mortalité semblait tout de même plus élevée dans le groupe «ATBproba - ». Mais les groupes n'étaient pas complètement comparable avec une proportion de sepsis sévère pré opératoire plus élevé dans ce groupe.

Etonnamment, 17 des 24 patients admis en réanimation n'avaient pas une antibiothérapie première efficace. Ce résultat est bien expliqué par le fait que nous nous sommes intéressés à la première antibiothérapie reçue, administrée le plus souvent aux urgences ou au bloc opératoire. De plus, tous les patients n'étaient pas admis en réanimation en post opératoire 
immédiat, certains présentant une aggravation secondaire, lors de leur hospitalisation en service de chirurgie.

Pour les patients de réanimation, donc présentant une forme grave de péritonite, l'antibiothérapie proposée par le Consensus de la SFAR est la Tazocilline, le plus souvent associé à un aminoside [1]. La supériorité de cette association par rapport à la Tazocilline seule est discutée [67]. Cette bêtalactamine, de spectre large, couvre l'essentiel des entérobactéries et des bacilles pyocyaniques communautaires, mais aussi l'entérocoque et les anaérobies. Les résistances à cet antibiotique sont de ce fait beaucoup plus rares $(83 \%$ d'efficacité dans notre étude). D'ailleurs, dans le protocole d'antibiothérapie probabiliste de la Réanimation Chirurgicale du CHU de Rouen, la Tazocilline en association à la Gentamycine représente l'antibiothérapie de première intention pour la péritonite communautaire grave.

Quant à la morbidité, la durée de ventilation, la durée d'hospitalisation en réanimation, la durée d'hospitalisation étaient d'autant plus longues que l'antibiothérapie probabiliste était inefficace, mais ces résultats étaient non significatifs, faute d'effectif plus grand très probablement. Néanmoins, beaucoup de séries ont bien fait le lien (significatif) entre antibiothérapie probabiliste inadaptée et morbité en réanimation [40,66].

Le siège de la péritonite peut influencer le devenir du patient. Dans notre série, la plupart des péritonites étaient d'origine sous-mésocolique avec une nette prédominance colique. Notre répartition était légèrement différente de celle habituellement retrouvée dans la littérature, les origines sus mésocoliques étant moins représentées [13, 14, 67]. La péritonite sus mésocolique d'origine biliaire est connue pour être plus sévère. Etant rare dans notre série (5\%), ceci peut aussi expliquer notre taux de mortalité plutôt bas (15\%) [39].

Parmi nos patients, la première cause des péritonites était d'origine appendiculaire (39\%), avec un taux similaire dans les 2 groupes «ATB proba + » et « ATB proba - ». La fréquence était relativement semblable aux données de la littérature [14, 39]. Dans notre sous groupe "péritonite appendiculaire », aucune mortalité, aucune hospitalisation secondaire en réanimation n'a été retrouvée et ce malgré $61 \%$ d'antibiothérapies probabilistes inefficaces. L'abcès était la principale complication post-opératoire ( 5 sur 39 , dont 4 dans le groupe «ATBproba - »), traitée avec succès par antibiotique seul dans 4 cas, et ayant nécessité un drainage per cutané sous contrôle radiologique dans 1 cas. D'un point de vue physiopathologique, ces abcès post-appendicectomie peuvent avoir deux causes. D'une part 
le lavage péritonéal, qui est impératif et qui doit être abondant, peut être entravé par une incision minime ou bien un abord par coeliochirurgie. D'autre part, une antibiothérapie initiale inadaptée malgré un traitement chirurgical optimal peut aussi causer ces abcès secondaires.

Ce dernier point souligne bien la complexité du choix de l'antibiothérapie probabiliste. En association avec un traitement chirurgical optimal, notre antibiothérapie vise-t-elle à couvrir l'ensemble des germes potentiellement retrouvés à la culture ? dont les entérocoques ? Aller dans ce sens nous exposerait à "surtraiter" inutilement un certain nombre de patients, avec toutes les conséquences économiques et écologiques qui en découlent. L'inverse augmenterait la morbidité sans augmenter la mortalité, y compris dans les formes peu graves, comme la péritonite appendiculaire. Ceci replace au centre du débat la nécessité absolue de traiter ou non l'ensemble des germes retrouvés à la culture du liquide péritonéal.

\section{Etude bactériologique}

La répartition des germes retrouvés dans notre étude était globalement comparable avec la littérature. Plus de la moitié étaient constituée par les entérobactéries (51\%), suivi par les cocci gram positif (26\%) et enfin par les anaérobies (15\%). Enterococcus sp représentait plus de $60 \%$ des cocci gram positif. De même, les bacilles Gram négatif non fermentants représentaient $8 \%$ de notre population contre 2\% dans l'étude de Dupont et coll. en 2000 et $5 \%$ dans celle de Montravers et coll. en 2009 [12, 14, 67]. Notre biais de sélection tendant à mettre en avant les résistances explique notre surrepésentation de ces 2 espèces naturellement résistante au C3G pour Enterococcus sp, et à l'Amox/Ac.clav pour Pseudomonas sp.

Les bactéries résistantes, cantonnées il n'y a pas si longtemps, au domaine hospitalier, apparaissent plus fréquemment en ville. Tous les domaines y sont confrontés, en urologie avec les infections urinaires, en pneumologie avec les pneumopathies infectieuses [24, 68] . Ces patients sont à risque d'être porteur de bactéries multirésistantes. Ils n'ont pas le profil des infections communautaires mais pas non plus le profil des infections nosocomiales. Les infections intra abdominales sont donc exposées à l'émergence des BLSE type CTX M et des carbapénèmases [26, 27].

Les médecins ont du s'adapter, élargissant le plus souvent le spectre de leur antibiothérapie à travers des associations thérapeutiques ou de nouvelles molécules.

Montravers et coll., en 2009, retrouve des profils de sensibilité différents avec ceux de notre 
travail, notamment une sensibilité d'E.coli à l'Amox/Ac.clav de 78\% contre seulement 35\%. Cette différence s'explique aussi par le même biais de sélection décrit précédemment et contre indique, de ce fait, toute extrapolation des profils de sensibilité à une population générale [14].

Cependant, pour les sensibilités aux autres antibiotiques, il est fort intéressant de constater que celles ci sont compatibles aux nôtres. Pour les germes autre que E.coli, toute comparaison reste limitée car notre travail n'étudie pas le même nombre de souches.

Les anaérobies ont le meme profil de résistance avec une sensibilité à l'Amox/Ac.clav de plus de $90 \%$, à la réserve près que Montravers et coll. s'intéresse plus particulièrement à Bactéroides sp [14].

En dehors des patients admis en réanimation, toutes les bactéries n'avaient pas forcément un antibiogramme. Le plus souvent, seul l'antibiogramme direct était rendu. Celui ci permet d'avoir un résultat rapide et adapté pour réorienter notre antibiothérapie. Tous les antibiotiques ne sont pas testés lors d'un antibiogramme direct. L'absence d'antibiogramme systématique et complet ne nous permet pas de répondre avec certitude à la question des sensibilités.

Cette stratégie est suffisante pour une activité clinique quotidienne, comme elle est pratiquée au CHU de Rouen depuis plusieurs années [34, 35].

Cependant, en réanimation, tous les germes sont documentés avec un antibiogramme complet. La fragilité des patients ne permet pas un retard d'efficacité thérapeutique.

\section{L'antibiothérapie}

Pour traiter une infection intra abdominale communautaire, il serait tentant d'élargir le spectre de l'antibiothérapie probabiliste. Une antibiothérapie trop large aurait dans un premier temps une efficacité certaine mais des conséquences écologiques bactériennes pouvant être catastrophiques à moyen et long termes : la pression de sélection engendrée par une telle attitude se traduirait rapidement par l'émergence de nouvelles résistances aux graves conséquences. L'industrie pharmaceutique fait preuve de moins d'innovation dans le développement des nouveaux antibiotiques que les bactéries dans l'émergence de nouvelles résistances. 
Dans notre étude, près de la moitié de l'antibiothérapie initiale ne répondait pas aux schémas proposés par le consensus français (45\%). Ce résultat est comparable à ceux de la littérature, retrouvant des taux d'adhésion aux protocoles de service variant de 42 à 50\% [69].

Les traitements administrés avaient soit un spectre trop large, soit au contraire un spectre trop restreint, soit une redondance dans leur activité antimicrobienne. Notons que l'imipénèm n'était retrouvé dans aucune prescription. Les raisons expliquant ce taux habituel mais bas sont multiples. L'absence de protocoles écrits dans le service ou le bloc opératoire, la surmédiatisation par les sociétés pharmaceutiques des bactéries multirésistantes peuvent contribuer à changer les schémas antibiotiques prescrits. Une autre hypothèse pourrait être avancée : l'allergie à la pénicilline. Dans notre étude, celle-ci n'a pas été recherchée. Cependant, parmi nos 99 patients, seuls 3 ont reçu des associations ne contenant pas de bêtalactamine. De plus, la SFAR en 2000 recommandait l'association aminoside Métronidazole chez le patient allergique, mais celle ci n'a jamais été retrouvée dans notre travail. Cette hypothèse de l'allergie ne peut donc pas expliquer à elle seule les "dérives" de prescription.

L'innovation individuelle dans l'antibiothérapie n'est pas toujours synonyme de succès thérapeutique. Seules 5 des associations thérapeutiques recensées avaient une sensibilité supérieure à $50 \%$. Mais ce résultat est biaisé, du fait de notre sélection de patients (qui présentaient à leur antibiogramme au moins une résistance à une des antibiothérapie du consensus).

Nous avons regardé dans notre travail l'efficacité théorique des différentes associations proposées par la SFAR sur nos 99 liquides péritonéaux. L'association Amox/ac.clav.Aminoside restait efficace à $62 \%$, l'aminoside permettant de majorer l'activité antibactérienne en cas d'E.Coli Amox/Ac.clav. I ou R, résistance de plus en plus retrouvée [61, 70].

Le Ticar/Ac.clav., d'utilisation moins fréquente, était tout aussi efficace, mais toujours en association avec les aminosides. Il présente l'avantage d'offrir une couverture sur les bacilles gram négatif fermentant de phénotype sauvage, aussi plus fréquemment retrouvés en ville à ce jour. Ces deux associations avaient l'atout de couvrir Enterococcus sp.

Les $\mathrm{C} 3 \mathrm{G}$, anciennes ou nouvelles, avaient une meilleure sensibilité sur les entérobactéries que l'Amox./Ac.clav. Le Métronidazole devait être systémtiquement ajouté pour être actif sur les anaérobies. Cependant elles présentaient l'inconvénient de ne pas couvrir Enterococcus sp. Le Céfépime, nouvelle céphalosporine, était plus souvent efficace que les anciennes. Celle ci a été beaucoup moins exposée, le développement de résistance bactérienne à cet antibiotique 
plus récent étant moins important.

Comme cité plus haut, la Tazocilline restait cependant très efficace grâce à son spectre large, avec un taux de résistance très faible (83\%). Un aminoside était systématiquement ajouté bien que cette association reste discutée en terme de supériorité [67].

Donc, en 2013, il nous semble que les recommandations de la SFAR restent toujours d'actualité, et notamment dans le cas des formes graves de péritonite communautaire.

Dans notre méthode, nous avons considéré Enterococcus $s p$ comme une espèce pathogène à part entière, alors que ce fait reste discuté, tout du moins dans les formes non sévères. La lecture critique des articles est difficile. Plusieurs études tendent à montrer l'absence de pathogénie, y compris des études récentes [2,71]. D'autres auteurs, à l'inverse, démontrent le rôle pathogène de ce cocci gram positif $[14,39,46,72,73]$.

L'enterocoque étant naturellement résistant aux céphalosporines et cet antibiotique étant proposées par la SFAR dans 3 des schémas thérapeutiques, le taux de résistance dans notre série est donc immanquablement majoré.

Notre étude ne montre pas de taux de complication plus importante en cas de première antibiothérapie inefficace (largement expliqué par la résistance de l'entérocoque aux $\mathrm{C} 3 \mathrm{G}$ ), laissant supposer un rôle peu pathogène de l'Entérocoque. Cependant, elle n'était pas construite pour établir un lien entre un Enterococcus sp non traité et la majoration des complications post opératoire des péritonites communautaires.

D'autres aspects de l'antibiothérapie impactent le devenir des patients présentant une péritonite communautaire.

Le délai d'instauration des antibiotiques est un point majeur de la prise en charge des patients septiques. Dans notre étude 49\% des patients avaient un délai d'instauration des antibiotiques inférieur à $6 \mathrm{~h}$. Contrairement à l'habitude, il n'est pas conseillé d'attendre la réalisation des prélèvements bactériologiques pour instaurer une antibiothérapie dans le but d'augmenter la sensibilité de la culture [19]. En effet, l'inoculum dans les péritonites est si important que la première dose d'antibiotiques ne peut pas négativer la culture du liquide péritonéal.

En cas de choc septique, on sait que chaque heure supplémentaire avant l'instauration d'un traitement antibiotique efficace, se traduit par un surcroit de décès de $7 \%$ [53]. Dans notre série, les patients admis en réanimation avaient un délai médian d'introduction des antibiotiques de $3 \mathrm{~h}$, délai très satisfaisant et d'ailleurs recommandé par la « surviving sepsis campaign » [66]. L'objectif de l'antibiothérapie précoce est de limiter les bactériémies et de 
réduire la fréquence des abcès résiduels [1, 61]. Peut-être ce délai court dans notre étude pourrait-il expliquer en partie le faible taux de complications post opératoires et de mortalité ?

Le guide du bon usage du médicamenteux impose une réévaluation à $72 \mathrm{~h}$ de l'antibiothérapie avec réadaptation, le plus souvent sous forme d'une dé-escalade $[74,75]$. Certes, seule la moitié de nos patients était traitée avec une antibiothérapie probabiliste efficace. Mais plus important, 13\% n'ont pas eu leur antibiothérapie réajustée alors qu'il l'aurait dû du fait d'un traitement probabiliste inefficace. Dans l'étude de Koperna, un accroissement de la mortalité était directement en lien avec l'absence de réadaptation de l'antibiothérapie [64].

Parmi ces patients il faut distinguer ceux dont l'antibiothérapie initiale était trop large, ciblée ou inefficace. Treize patients $(13,1 \%)$ avait une antibiothérapie initiale infructueuse non réadaptée.

Dans notre travail, parmi ces 13 patients, aucun n'a présenté de complication quand la cause était d'origine appendiculaire. A l'inverse, 2 des 3 patients admis en réanimation n'ayant pas une antibiothérapie adaptée à J4 étaient décédés. Aucun calcul statistique n'a pu être réalisé. De nombreux facteurs confondants peuvent expliquer ce résultat. Mais celui ci, malgré son faible effectif (3 patients) est bien corrélé à la littérature et conforte les stratégies du Surviving Sepsis Campaign [23].

\section{Prise en charge chirurgicale}

En plus de l'antibiothérapie efficace et administrée tôt, l'autre grand principe de la prise en charge des péritonites est la réalisation de la chirurgie dans un délai court.

Dans notre série, les patients étaient admis au bloc opératoire le plus souvent dans les $12 \mathrm{~h}$ (54,5\%), jusq'à $24 \mathrm{~h}$ pour $75 \%$ des patients. Ce délai précoce démontre sûrement la volonté de l'équipe de viscérale du CHU de Rouen, la bonne disponibilité des blocs opératoires et des équipes. En effet, au sein du CHU, 3 salles d'opération réunies dans un bloc d'urgences sont dédiées aux urgences nuit et jour, adossées à une salle de réveil 24/24. La filière de soins du syndrome abdominal aigu chirurgical semble donc efficace.

Les propositions du Surviving Sepsis Campaign recommandent en cas de sepsis sévère ou de choc septique une chirurgie dans les $12 \mathrm{~h}$ suivant le diagnostic. Les patients admis en 
réanimation avaient un délai « chirurgical » médian de $3 \mathrm{~h}$.

Prouvée à de nombreuses reprises dans la littérature, un traitement chirurgical satisfaisant et complet par un opérateur expérimenté a un impact direct sur la mortalité et sur les complications post opératoires des péritonites [7, 37, 76]. Des études anciennes rapportaient que, malgré une antibiothérapie efficace, le taux de mortalité était de $90 \%$ quand la source n'était pas contrôlée chirurgicalement.

Dans notre travail, la qualité du geste chirurgical n'a pas été évaluée, n'étant pas de la spécialité et du fait du caractère rétrospectif de notre analyse.

\section{Quelles solutions?}

A la vue de ces résultats, plusieurs pistes d'amélioration de la prescription d'antibiotiques sont envisageables.

L'informatisation des prescriptions, en cours de déploiement au CHU de Rouen, permettra de connaître systématiquement les durées d'antibiothérapie, de mieux visualiser par un code couleur ou autre, si celle ci est empirique ou adaptée, voire de bloquer la prescription « antibiotiques » à $72 \mathrm{~h}$ en l'absence de réévaluation de la stratégie antibactérienne.

La mise en place de protocoles écrits d'antibiothérapie probabiliste efficace et connus de tous au sein des blocs opératoires et des services est une mesure recommandée $[1,2,19]$. Elle limite les schémas thérapeutiques plus individuels, le plus souvent moins efficace [77].

L'appel par le médecin bactériologiste permet d'alerter le praticien en cas de bactérie au profil de résistance atypique. Cette pratique, déjà habituelle au CHU de Rouen, devrait sûrement être généralisée.

Le contrôle de la délivrance des antibiotiques par le pharmacien, après validation de l'indication et analyse de l'antibiogramme, est une mesure visant à réduire la prescription large. Ceci est mis en place dans notre CHU depuis plusieurs années. Même si cette mesure est jugée contraignante par la plupart des prescripteurs d'antibiotiques, elle a au moins le mérite d'imposer une rigueur de prescription, tant sur l'indication, sur le choix raisonné de l'antibiotique, le recours obligatoire à un référent bactériologique ou infectieux. De même, elle impose une ré évaluation à $72 \mathrm{~h}$.

Habituelle en réanimation, la tenue de staff multidisciplinaire de bactériologie dans les 
services de chirurgie permettrait de plus de solutionner les antibiothérapies problématiques et de mieux rétrocéder.

Aussi, on pourrait imaginer qu'au sein des services de chirurgie viscérale, plus vastes qu'un service de réanimation, des praticiens deviennent référents en antibiothérapie.

Ainsi, de nombreuses mesures sont possibles, plus ou moins réalisables, mais pouvant offrir à court terme, une optimisation de la prescription des antibiotiques. 


\section{Conclusion}

En dehors des cas graves de péritonite communautaire, une erreur sur l'antibiothérapie initiale ne semble pas grevée d'une sur-mortalité. Cependant, elle peut majorer les complications chirurgicales et médicales post opératoires. Cette constatation souligne bien la nécesssité absolue d'obtenir un traitement chirurgical complet.

Néanmoins, l'antibiothérapie probabiliste détient un rôle majeur chez le patient fragilisé de réanimation. Même si nos résultats sur la mortalité ne montrent pas de différence significative dans notre sous-groupe «réanimation », la majoration de la morbi-mortalité due à une antibiothérapie probabiliste inadaptée n'est plus à démontrer dans la littérature.

Certes, le choix de l'antibiothérapie probabiliste est capital, mais d'autres points de prise en charge sont essentiels, comme la réduction du délai d'administration de la première dose, la réévaluation, la notion de référentiel de service.

Malgré un biais de sélection majeur de nos patients pour lesquels les bactéries résistantes étaient sur-représentées, il nous semble que les antibiothérapies proposées par la SFAR restent toujours d'actualité.

Les recommandations américaines, plus récentes, recommandent l'usage beaucoup plus large en probabiliste de molécules à large spectre comme les carbapénèmes. Seulement, l'écologie bactérienne nord-américaine est différente, suite, entre autres, à une politique de gestion des antibiotiques différente.

En France, le recours systématique à ces molécules en probabiliste paraît à ce jour prématuré. Sachons garder nos nouveaux antibiotiques. Ils sont une denrée rare. A l'image de l'eau potable dans le monde, ce qui est rare doit être utilisé avec parcimonie, précision et efficacité. 


\section{Bibliographie}

1. Prise en charge des péritonites comunautaires - conference de consensus - texte court. Société Française d'Anesthésie-Réanimation.Ann Fr Anesth Reanim 2001;20:368s$73 \mathrm{~s}$.

2. Solomkin JS, M.J., Bradley JS et al., Diagnosis and management of complicated intra-abdominal infection in adults and children: guidelines by the Surgical Infection Society and the Infectious Diseases Society of America. Surg Infect (Larchmt). 11:79109, 2010.

3. Hall JC, H.K., Papadimitriou JM, Platell C, The pathobiology of peritonitis. Gastroenterology 1998;114:185-96.

4. Nakatani T, T.S., Mizukami S, Okamoto K, Shiraishi Y, Nakamura T., Peritoneal lymphatic stomata of the diaphragm in the mouse: process of their formation. Anat Rec. 1997 May;248(1):121-8.

5. Andersson R, T.K., Alwmark A, Bengmark S., Factors influencing the outcome of E. coli peritonitis in rats. Acta Chir Scand. 1989 Mar;155(3):155-7.

6. $\quad$ GL., S., Intestinal flora in health and disease. Gastroenterology. 1984 Jan;86(1):17493.

7. Seiler CA, B.L., Forssmann U, Baer HU, Büchler MW., Conservative surgical treatment of diffuse peritonitis. Surgery. 2000 Feb;127(2):178-84.

8. Wittmann., D., Intraabdominal infection. Marcel Dekker Inc Ed. Frankfurt 1991. pp 8.

9. Onderdonk AB, W.W., Sullivan NM, Bartlett JG, Gorbach SL. , Experimental intraabdominal abscesses in rats: quantitative bacteriology of infected animals. Infect Immun. 1974 Dec;10(6):1256-9.

10. Dupont H, C.C., Carlet J., The severe generalized peritonitis study group. Monotherapy with a broad-spectrum beta-lactam is as effective as its combination with an aminoglycoside in treatment of severe generalized peritonitis: a multicenter randomized controlled trial. Antimicrob Agents Chemother 2000;44:2028-33.

11. Montravers P, C.A., Gauzit R, Lepape A, Marmuse JP, Vouillot C, et al. , Clinical and therapeutic features of non postoperative nosocomial intra-abdominal infections. Ann Surg 2004; 239: 409-16.

12. Roehrborn A, T.L., Potreck O, Ebener C, Ohmann C, Goretzki PE, et al., The microbiology of postoperative peritonitis. Clin Infect Dis 2002;33:1513-9.

13. Gauzit R, P.Y., Barth X, Mistretta F, Lalaude O; Top Study Team Epidemiology, management, and prognosis of secondary non-postoperative peritonitis: a French prospective observational multicenter study. Surg Infect (Larchmt). 2009 Apr;10(2):119-27.

14. Montravers P, L.A., Dubreuil L, et al, Clinical and microbiological profiles of community-acquired and nosocomial intra-abdominal infections: results of the French prospective, observational EBIIA study. J Antimicrob Chemother 63: 785-94, 2009.

15. Cooper GS, S.D., Salata RA, Intraabdominal infection: differences in presentation and outcome between younger patients and the elderly. Clin Infect Dis 1994;19:146-8.

16. Pacelli F, D.G., Alfieri S, Piccioni E, Sgadari A, Gui D, et al., Prognosis in intraabdominal infections. Multivariate analysis on 604 patients. Arch Surg 1996;131:6415.

17. Ohmann C, W.D., Wacha H., Prospective evaluation of prognostic scoring systems in peritonitis. Peritonitis Study Group. EurJ Surg 1993;159:267-74.

18. DH., W., Intraabdominal infection. World J Surg 1990;14:145-7. 
19. Montravers P, M.F., Cargeac A Péritonites. In: EMC Anesthésie-Réanimation. Elsevier Masson SAS, 36-726-A-30, 2005.

20. Onderdonk AB, B.J., Louie T, Sullivan-Seigler N, Gorbach SL. , Microbial synergy in experimental intra-abdominal abscess.Infect Immun 1976;13:22-6.

21. Coenen S, M.A., Adriaenssens N, Vankerckhoven V, Hendrickx E, Goossens H; ESAC Project Group., European Surveillance of Antimicrobial Consumption (ESAC): outpatient parenteral antibiotic treatment in Europe.J Antimicrob Chemother. 2009 Jul;64(1):200-5.

22. pratiques, C.s.c.e.S.c.d.p.i.n.e.a.e.v.n.i.s.l.s.a.s.e.a., Recommandations relatives aux mesures à mettre en coeuvre pour prévenir l' 'émergence des entérobactéries BLSE et lutter contre leur dissémination. Février 2010.

23. Marc LEONE - Ioanna BOYADJIEV - Claude MARTIN, Péritonites communautaires: quelle antibiothérapie probabiliste? URGENCE PRATIQUE 2007 No80.

24. Rodriguez-Bano J, N.M., Romero L, Martinez-Martinez L, Muniain MA, Perea EJ, Perez-Cano R, Pascual A., Epidemiology and clinical features of infections caused by extended-spectrum beta-lactamase-producing Escherichia coli in nonhospitalized patients. J. Clin. Microbiol. 2004, 42 : 1089-94.

25. Paterson DL, B.R., Extended-spectrum beta-lactamases: a clinical update. Clin Microbiol Rev. 2005 Oct;18(4):657-86.

26. Cantón R, A.M., Carmeli Y et al., Rapid evolution and spread of carbapenemases among Enterobacteriaceae in Europe. Clinical Microbiology and Infection: The Official Publication of the European Society of Clinical Microbiology and Infectious Diseases. 18: 413-31, 2012.

27. Kang C-I, W.Y., Lee MY et al., Epidemiology and risk factors of community onset infections caused by extended-spectrum $\beta$-lactamase-producing Escherichia coli strains. J. Clin. Microbiol. 50: 312-7, 2012.

28. Marco CA, S.C., Keyl PM, Menkes ED, Doehring MC., Abdominal pain in geriatric emergency patients: variables associated with adverse outcomes. Acad Emerg Med. 1998 Dec;5(12):1163-8.

29. Moriwaki Y, S.M., Toyoda H, Kosuge T, Arata S, Iwashita M, Tahara Y, Suzuki N. , Ultrasonography for the diagnosis of intraperitoneal free air in chest-abdominalpelvic blunt trauma and critical acute abdominal pain. Arch Surg. 2009 Feb;144(2):137-41.

30. BM, R., Evaluation of procalcitonin for predicting septic multiorgan failure and overall prognosis in secondary peritonitis: a prospective, international multicenter study. Arch Surg. 2007 Feb;142(2):134-42.

31. HB, R., Procalcitonin (PCT) in patients with abdominal sepsis. Intensive Care Med. 2000 Mar;26 Suppl 2:S165-9.

32. AR, N., Procalcitonin ratio indicates successful surgical treatment of abdominal sepsis. . Surgery. 2009 Jan;145(1):20-6.

33. Trullen, V., Prospective study of procalcitonin as a diagnostic marker of the severity of secondary peritonitis. Cir Esp. 2009 Jul;86(1):24-8.

34. Hansen, D.S., Jensen, A.G., Norskov-Lauritsen, N., Skov, R., and B. Bruun, Direct identification and susceptibility testing of enteric bacilli from positive blood cultures using Vitek (GNI+GNS-GA). Clin. Microbiol. Infect. 8:38-34, 2002.

35. Noussair, L., and M.-H. Nicolas-Chanoine, Obtention of bacterial antibiotic susceptibility following standard conditions directly from infected biological fluids and positive blood cultures. Pathol. Biol. 55:143-148 2007.

36. JG., B., Intra-abdominal sepsis. Med Clin North Am 1995;79: 599-617. 
37. Bohnen J, B.M., Meakins J, McLean P., Prognosis in generalized peritonitis: relation to cause and risk factors. Arch Surg 1983;118:285-90.

38. Koperna T, S.F., Relaparotomy in peritonitis: prognosis and treatment of patients with persisting intraabdominal infection. World J Surg. 2000 Jan;24(1):32-7.

39. Riché FC, D.X., Laisné MJ, et al., Factors associated with septic shock and mortality in generalized peritonitis: comparison between community-acquired and postoperative peritonitis. Crit Care 13: R99, 2009.

40. Mosdell DM, M.D., Voltura A, Pitcher DE, Twiest MW, Milne RL, et al. , Antibiotic treatment for surgical peritonitis. Ann Surg 1991;214:543-9.

41. Carlet, L'infection en réanimation. 1986 p. pp126-138.

42. Pitcher WD, M.D., Critical importance of early diagnosis and treatment of intraabdominal infection. Arch Surg 1982;117:328-33.

43. Dellinger RP, L.M., Carlet JM, Bion J, Parker MM, Jaeschke R, et al. , Surviving Sepsis Campaign: International guidelines for mana- gement of severe sepsis and septic shock. Crit Care Med 2008;36: 296-327.

44. Bartlett JG, O.A., Louie T, Kasper DL, Gorbach SL., Lessons from an animal model of intra-abdominal sepsis. Arch Surg 1978;113: 853-7.

45. Dougherty, S.H., Antimicrobial culture and susceptibility testing has little value for routine management of secondary bacterial peritonitis. Clin Infect Dis 1997 ; 25: S258-61.

46. Burnett RJ, H.D., Dellinger EP, Reinhart HH, Bohnen JM, Rotstein OD, et al. , Definition of the role of enterococcus in intraabdominal infection: analysis of a prospective randomized trial. Surgery 1995;118:716-21.

47. Weigelt JA, E.S., Thal ER, Palmer LD, Newman VS. , Abdominal surgical wound infection is lowered with improved perioperative enterococcus and bacteroides therapy. J Trauma 1993;34:579-85.

48. Montravers P, D.H., Gauzit R et al. , Candida as a risk factor for mortality in peritonitis. Crit. Care Med. 34: 646-52, 2006.

49. Dupont H, P.-B.C., Muller-Serieys C et al., Predictive factors of mortality due to polymicrobial peritonitis with Candida isolation in peritoneal fluid in critically ill patients. Arch Surg. 137: 1341-1346, 2002.

50. RR., M., Comparative penetration of amikacin, gentamicin, and penicillin $G$ into exudate fluid in experimental sterile peritonitis. Antimicrob Agents Chemother 1977;11:110-3.

51. Berglund JA, A.L., Skau T, Nyström PO., Host defence and bacterial growth in fosfomycin-treated peritonitis. Acta Chir Scand 1990;156:529-36.

52. JC, P., Les suppurations abdominales : point de vue d'un infectiologue. Ann Chir 1985;39:55-60.

53. Kumar A, R.D., Wood KE, Light B, Parrillo JE, Sharma S, et al. , Duration of hypotension before initiation of effective antimicrobial therapy is the critical determinant of survival in human septic shock. Crit Care Med 2006;34:1589-96.

54. C. Eckmann, M.d., P. Montravers, R. Kozlov, G. sganga, Antimicrobial treatment of complicated Intra-abdominal infections and the new IDSA guIdelines - A commentary and an alternatIve European Approach according to clinical definitions. Eur J Med Res 16: 115-126, 2011.

55. Schneider CP, S.C., Vilsmaier M, Küchenhoff H, Hofner B, Jauch KW, Hartl WH., Prognostic factors in critically ill patients suffering from secondary peritonitis: a retrospective, observational, survival time analysis. World J Surg. 2009 Jan;33(1):3443. 
56. Koperna T, S.F., Prognosis and treatment of peritonitis. Do we need new scoring systems? Arch. Surg. 1996, 131: 180-186.

57. Montravers P, G.R., Muller C, et al., Emergence of antibiotic-resistant bacteria in cases of peritonitis after intraabdominal surgery affects the efficacy of empirical antimicrobial therapy. Clin Infect Dis 23: 486-94, 1996.

58. Sotto A, L.J., Fabbro-Peray P, Muller L, Tafuri J, Navarro F, Prudhomme M, De La Coussaye JE, Evaluation of antimicrobial therapy management of 120 consecutive patients with secondary peritonitis. J Antimicrob Chemother. 2002 Oct;50(4):569-76.

59. Hopkins, J.A., J.C. Lee, and S.E. Wilson, Susceptibility of intra-abdominal isolates at operation: a predictor of postoperative infection. Am Surg, 1993. 59(12): p. 791-6.

60. Falagas, M.E., et al., Risk factors leading to clinical failure in the treatment of intraabdominal or skin/soft tissue infections. Eur J Clin Microbiol Infect Dis, 1996. 15(12): p. 913-21.

61. T. Clavier, p.G., b. Veber, Péritonites communautaires. Réanimation Médicale 2012 ; p 235-249.

62. Knaus WA, D.E., Wagner DP, Zimmerman JE, APACHE II: a severity of disease classification system. Crit Care Med 1985;13:818-29.

63. Le Gall JR, L.S., Saulnier F, A new simplified acute physiology score (SAPSII) based on a European-North American multicenter study. JAMA 1993;270:29057-68.

64. Koperna, T. and F. Schulz, Prognosis and treatment of peritonitis: Do we need new scoring systems? Archives of Surgery, 1996. 131(2): p. 180-186.

65. Ibrahim EH, S.G., Ward S, Fraser VJ, Kollef MH, The influence of inadequate antimicrobial treatment of bloodstream infections on patient outcomes in the ICU setting. Chest. 2000 Jul;118(1):146-55.

66. Dellinger RP, L.M., Rhodes A, Annane D, Gerlach H, Opal SM, Sevransky JE, Sprung CL, Surviving sepsis campaign: international guidelines for management of severe sepsis and septic shock: 2012. Crit Care Med. 2013 Feb;41(2):580-637.

67. Dupont H, C.C., Carlet J Monotherapy with a broad-spectrum beta- lactam is as effective as its combination with an aminoglycoside in treatment of severe generalized peritonitis: a multicenter randomized controlled trial. The Severe Genera-lized Peritonitis Study Group. Antimicrob Agents Chemother 44: 2028-33, 2000.

68. SolomkinJ, T., GrahamDR,GesserRM,MeibohmAR,RoyS, WoodsGL., Treatmentofpolymicrobialinfections:posthocanalysis of three trials comparing ertapenem and piperacillin tazobactam.J. Antimicrob. Chemother. 2004, 53 Suppl 2 : ii51-57.

69. B.favier, audits des pratiques d'antibioprophylaxie en chirurgie.

70. Ortega A, O.J., Aranzamendi-Zaldumbide M, et al., Epidemiology and resistance mechanisms to amoxicillin-clavulanate in Escherichia coli: A Spanish mul-ticenter study. Antimicrobial Agents and Chemotherapy., 2012

71. Namias N, S.J., Jensen EH, et al., Randomized, multicenter, double- blind study of efficacy, safety, and tolerability of intravenous ertapenem versus piperacillin/tazobactam in treatment of complicated intra-abdominal infections in hospitalized adults. Surg Infect (Larchmt) 8: 15-28, 2007

72. Cercenado E, T.L., Cantón R, et al., Multicenter study evaluating the role of enterococci in secondary bacterial peritonitis. J Clin Microbiol 48: 456-9, 2010.

73. Sitges-Serra A, L.M., Girvent M, Almirall S, Sancho JJ., Postoperative enterococcal infection after treatment of complicated intra-abdominal sepsis. Br J Surg. 2002 Mar;89(3):361-7. 
74. Conférence de consensus. Prévention des infections à bactéries multirésistantes en réanimation (en dehors des modalités d'optimi- sation de l'antibiothérapie). Paris 1996.

75. $\quad$ consensus., C.r.d., Comment améliorer la qualité de l'antibiothérapie dans les établissements de soins? Med Mal Infect 2002;32:320-8.

76. Pitcher WD, M.D., Critical importance of early diagnosis and treatment of intraabdominal infection. Arch Surg 1982;117:328-33.

77. Philippe Montravers, M.D., Pascal Augustin, Optimisation de l'antibiothérapie des péritonites. MAPAR 2009 ; 219-224. 